\title{
The development and application of two-time-scale turbulence models for non-equilibrium flows
}

DOI:

10.1016/j.ijheatfluidflow.2018.04.010

\section{Document Version}

Accepted author manuscript

Link to publication record in Manchester Research Explorer

\section{Citation for published version (APA):}

Klein, T., Craft, T., \& lacovides, H. (2018). The development and application of two-time-scale turbulence models for non-equilibrium flows. International Journal of Heat and Fluid Flow, 71, 334-352.

https://doi.org/10.1016/j.ijheatfluidflow.2018.04.010

\section{Published in:}

International Journal of Heat and Fluid Flow

\section{Citing this paper}

Please note that where the full-text provided on Manchester Research Explorer is the Author Accepted Manuscript or Proof version this may differ from the final Published version. If citing, it is advised that you check and use the publisher's definitive version.

\section{General rights}

Copyright and moral rights for the publications made accessible in the Research Explorer are retained by the authors and/or other copyright owners and it is a condition of accessing publications that users recognise and abide by the legal requirements associated with these rights.

\section{Takedown policy}

If you believe that this document breaches copyright please refer to the University of Manchester's Takedown Procedures [http://man.ac.uk/04Y6Bo] or contact uml.scholarlycommunications@manchester.ac.uk providing relevant details, so we can investigate your claim.

\section{OPEN ACCESS}




\title{
The Development and Application of Two-Time-Scale Turbulence Models for Non-Equilibrium Flows
}

\author{
T. S. Klein ${ }^{1}$, T. J. Craft and H. Iacovides \\ School of Mechanical, Aerospace and Civil Eng., The University of Manchester, UK \\ ${ }^{1}$ Currently at: Chemical Eng. Depart., Federal University of Rio de Janeiro, Brazil \\ tania@eq.ufrj.br
}

April 25, 2018

\begin{abstract}
This study re-visits the largely overlooked, but highly promising, topic of multi-scale RANS modelling for non-equilibrium turbulent flows. The paper presents the development of RANS models which, at an effective-viscosity level, involve two transport equations for the turbulent kinetic energy; one for the energy of the large scale, energy-producing, eddies and one for that of the smaller eddies, which, through continuous break-up, transfer turbulence energy to the dissipative scales. Two transport equations for the transfer-rate of the turbulent kinetic energy are also necessary, one for the rate of energy transfer from the large to the smaller scales and one for the rate of transfer from the smaller to the dissipative scales, the latter being of course the dissipation rate of turbulence. The two-time-scale model of Hanjalic et al. (1980) has been the starting point of the current developments. The coefficients of the terms which appear in these models have been determined from asymptotic analyses of decaying grid turbulence, homogeneous shear flows and local-equilibrium boundary-layer flows. The models were subsequently further developed to become more responsive to strong non-equilibrium features, such as those caused by strong shear. It has been identified that in general, models which have been optimised to satisfy equilibrium flows (as is usually done) are unable to capture the strong changes the flows are subjected to due to highly non-equilibrium effects, thus needing further development. Within the two-time-scale framework, it has been found possible to overcome this problem, by exploiting the additional information available on the turbulence spectrum. In a further departure from the development of earlier effective-viscosity two-time scale models, here the eddy viscosity expression is made sensitive to the local strain rate, as was also the case in single-scale models developed by Craft et al. (1996).

The original contribution of this research is the development of two widely tested two-timescale linear-eddy-viscosity models, referred to here as NT1 and NT2. The difference between these two models is primarily in the eddy viscosity formulation. The two resulting models have been tested over nine test cases and their performance has been compared to those of the standard high-Reynolds number $k-\varepsilon$ model, the SSG Reynolds stress transport model and the two-time-scale model of Hanjalic et al. (1980). The test cases comprise of homogeneous shear and normally strained flows, adverse-pressure-gradient, favourable-pressure-gradient and oscillatory boundary layer flows, fully developed oscillatory and ramp up pipe flows and steady and pulsated backward-facing step flows. The models proposed here show considerable promise. They perform well in all cases tested and provide clear improvements over a range of single- and multi-scale models tested in an earlier study (Klein et al., 2015), over a set of test cases which cover a wide range of physical flow phenomena. Moreover, these improvements are achieved without any significant increase in computational requirements in comparison to the computational needs of single-time-scale eddy-viscosity models.
\end{abstract}

\section{Introduction}

Most turbulence models used in Computational Fluid Dynamics (CFD), within the ReynoldsAveraged-Navier-Stokes (RANS) framework, can be classified as Single-Time-Scale (STS) models. 
The reason for that is the assumption behind the development process of these models, which considers that the flow can be characterized by a single time or length scale. However, this assumption is, admittedly, an approximation of the real physics of the flows, since the turbulent kinetic energy is characterized by a wide range of length (or time) scales, which build the so called turbulent kinetic energy spectrum.

The turbulent kinetic energy spectrum represents the vortex stretching and cascade processes typical of turbulent motions. These phenomena become therefore important enough to be taken into account, when non-equilibrium features are present in the flow. Non-equilibrium flows include flows subjected to rapid changes, such as sudden expansions and/or contractions, rapid variations in time and/or imposed pressure gradients. Such conditions are very often found in industrial flows, which in turn emphasizes the importance of developing models that can reliably predict non-equilibrium flows. As STS models assume spectral equilibrium in order to describe the flow through a single time or length scale, they are thus not expected to be capable of reproducing such non-equilibrium features, especially capturing the lag in response of the turbulence field to changes in the mean flow field.

In order to take into account some features of the turbulent kinetic energy spectrum, MultipleTime-Scale (MTS) models arose within the RANS framework. For practical purposes, and for the sake of simplicity and reduced computational effort, these models usually employ two time (or length) scales to characterize the flow and are therefore referred to as Two-Time-Scale (TTS) models.

Recent studies of the authors (Klein, 2012; Klein et al., 2015) have investigated the performance of some TTS models against commonly used and classical STS models in a wide range of 2-D nonequilibrium flows. Both eddy viscosity and Reynolds stress transport schemes were considered for both STS and TTS models. The results confirmed the expectation of possible improvements in predicting non-equilibrium flows by the TTS models, as also indicated by Hanjalic et al. (1980), Wilcox (1988), Kim and Chen (1989), Kim (1991), Chen and Guo (1991), Kim and Benson (1992), Duncan et al. (1993), Nagano et al. (1997), Rubinstein (2000), Stawiarski and Hanjalic (2002), Cadiou et al. (2004) and Stawiarski and Hanjalic (2005) who either developed or used TTS models to predict non-equilibrium flows. The aforementioned works comprised different features of turbulence modelling, such as eddy viscosity and Reynolds stress transport schemes and low Reynolds number approaches. Klein (2012) and Klein et al. (2015) also identified the lack of a turbulence model that performed consistently well in all flow cases tested.

These results thus motivated the present work where two two-time-scale linear-eddy-viscosity models have been developed. The objective has been, by introducing the effective modelling principles identified in our earlier survey of single- and two-scale models, Klein et al. (2015), to develop two-time scale models which can be used to predict non-equilibrium flows reliably. In this paper the main steps which led to the final form of the models are described and their performances are evaluated in several 2-D non-equilibrium flows against the performance of three other models: the standard $k-\varepsilon$ model of Jones and Launder (1972) and Launder and Spalding (1974), the SSG Reynolds stress transport model of Speziale (1991) and the TTS linear-eddy-viscosity model of Hanjalic et al. (1980).

\section{Turbulence Modelling}

The development of the MTS models proposed here used the linear-eddy-viscosity two-time-scale model proposed by Hanjalic et al. (1980) as the starting point. This model is often used as a reference for multiple-time-scale approaches.

The modelling framework Hanjalic et al. (1980) proposed consisted of splitting the turbulent kinetic energy spectrum into three parts: a production zone, a transfer zone and a dissipation zone. Following the vortex stretching and cascade processes, the generation of turbulence occurs in the large eddies in the production zone. The turbulent kinetic energy is then transfered to smaller eddies, through eddy break-up, in the transfer zone until the eddies become small enough 
for viscosity to convert their kinetic energy into heat, in the dissipation zone. The model thus suggests that a variable $k_{P}$ denotes the part of the total turbulent kinetic energy $k$ which is stored in the large eddies in the production zone and that $k_{T}$ represents the part of the total turbulent kinetic energy which is stored in smaller eddies in the transfer zone. The turbulent kinetic energy is transfered from the production to the transfer zone at a rate $\varepsilon_{P}$ and from the transfer to the dissipation zone at a rate $\varepsilon_{T}$. However, no part of the turbulent kinetic energy is stored in the dissipation zone because it is assumed that it is immediately dissipated into heat due to spectral equilibrium between these two zones, that is, $\varepsilon_{T}=\varepsilon$, the latter being the eddy dissipation rate usually used in single-time-scale models. The total turbulent kinetic energy can thus be expressed as $k=k_{P}+k_{T}$.

When the framework above is applied to a linear-eddy-viscosity scheme, four transport equations are needed, one for each partition variable $\left(k_{P}, k_{T}, \varepsilon_{P}\right.$ and $\left.\varepsilon_{T}\right)$. Whereas in STS models the eddy viscosity is given by $\mu_{t}=\rho c_{\mu} \frac{k^{2}}{\varepsilon}$, in TTS models, more options are available, some of which will be explored later. The Reynolds stresses are still modelled through the linear effective-viscosity approximation:

$$
\overline{u_{i} u_{j}}=-\nu_{t}\left(\frac{\partial U_{i}}{\partial x_{j}}+\frac{\partial U_{j}}{\partial x_{i}}\right)+\frac{2}{3} k \delta_{i j}
$$

In spite of the inherent limitations of linear-eddy-viscosity models, such simple schemes allow one to identify the improvements associated with the presence of spectral terms. For this reason, this study focuses on linear-eddy-viscosity two-time-scale models. Besides this, due to their simplicity, robustness and capacity to provide relatively reasonable results for a wide range of flows, lineareddy-viscosity approaches are the most widely used models and improvements in such schemes should therefore be useful for practical purposes.

General transport equations for the partition variables, proposed by Hanjalic et al. (1980), can be expressed as:

$$
\begin{gathered}
\frac{D k_{P}}{D t}=P_{k}-\varepsilon_{P}+\frac{\partial}{\partial x_{j}}\left[\left(\nu+\frac{\nu_{t}}{\sigma_{k_{P}}}\right) \frac{\partial k_{P}}{\partial x_{j}}\right] \\
\frac{D k_{T}}{D t}=\varepsilon_{P}-\varepsilon_{T}+\frac{\partial}{\partial x_{j}}\left[\left(\nu+\frac{\nu_{t}}{\sigma_{k_{T}}}\right) \frac{\partial k_{T}}{\partial x_{j}}\right] \\
\frac{D \varepsilon_{P}}{D t}=C_{P 1} P_{k} \frac{\varepsilon_{P}}{k_{P}}-C_{P 2} \frac{\varepsilon_{P}^{2}}{k_{P}}+\frac{\partial}{\partial x_{j}}\left[\left(\nu+\frac{\nu_{t}}{\sigma_{\varepsilon_{P}}}\right) \frac{\partial \varepsilon_{P}}{\partial x_{j}}\right]+C_{P 1}^{\prime} k_{P} \frac{\partial U_{l}}{\partial x_{m}} \frac{\partial U_{i}}{\partial x_{j}} \epsilon_{l m k} \epsilon_{i j k} \\
\frac{D \varepsilon_{T}}{D t}=C_{T 1} \frac{\varepsilon_{P} \varepsilon_{T}}{k_{T}}-C_{T 2} \frac{\varepsilon_{T}^{2}}{k_{T}}+\frac{\partial}{\partial x_{j}}\left[\left(\nu+\frac{\nu_{t}}{\sigma_{\varepsilon_{T}}}\right) \frac{\partial \varepsilon_{T}}{\partial x_{j}}\right]
\end{gathered}
$$

where $P_{k}$ stands for the turbulent kinetic energy production term: $P_{k}=-\overline{u_{i} u_{j}} \frac{\partial U_{i}}{\partial x_{j}}$.

These equations have been the starting point for the modelling task in this work. The only non-standard term above is the last term in equation (4) which was introduced by Hanjalic et al. (1980) in order to improve the sensitivity of the model to normal straining. This term has also been studied here and because of this it has been retained in the general equations above.

Consistently with the practical purpose of the proposed models, they are developed here to be used together with wall functions. Although wall functions are not suitable to solve the viscous sublayer, they are widely used in industrial applications, since they allow coarser meshes in near-wall regions and therefore require less computational efforts. In the results presented below the same wall functions have been used with all the models tested, thus providing a consistent comparison of the model performances outside the viscous layers. The viscous diffusion terms in equations (2) to (5) have been kept though, because they belong to the exact $k$ and $\varepsilon$ equations (and therefore to $k_{P}, k_{T}, \varepsilon_{P}$ and $\varepsilon_{T}$ 's) and, although their impact on results will be negligible here, they can have a beneficial effect on numerical stability in regions of very low turbulence. 
The modelling process in this work starts by applying the above equations to asymptotic analysis of homogeneous shear flows, decaying grid turbulence and local equilibrium boundary layer flows. These are standard type of solutions which can be found in turbulent flow theory (for example, Pope (2000) and Tennekes and Lumley (1972)) and have also been used for turbulence model tuning in a number of works (Kim and Benson, 1992; Nagano et al., 1997; Stawiarski and Hanjalic, 2002; Cadiou et al., 2004; Stawiarski and Hanjalic, 2005). This process results in a set of constraint equations which ensure that the models return the expected behaviour in these basic standard cases. This procedure is usually adopted in the development of both STS and MTS models, however, the tuning of the coefficients is here completed through numerical experiments for homogeneous shear flows for a wide range of dimensionless shear $\eta$, which is defined as: $\eta=\max \left[\frac{k}{\varepsilon_{T}} \sqrt{\frac{1}{2} S_{i j} S_{i j}}, \frac{k}{\varepsilon_{T}} \sqrt{\frac{1}{2} W_{i j} W_{i j}}\right]$, $S_{i j}$ being the mean strain given by $S_{i j}=\frac{\partial U_{i}}{\partial x_{j}}+\frac{\partial U_{j}}{\partial x_{i}}$ and $W_{i j}$ being the mean vorticity given by $W_{i j}=\frac{\partial U_{i}}{\partial x_{j}}-\frac{\partial U_{j}}{\partial x_{i}}$. In homogeneous shear flows $\eta$ reduces to $\eta=\frac{k}{\varepsilon_{T}} \frac{d U}{d y}$. Following the practice successfully introduced by Craft et al. (1996) and subsequently further refined by Craft et al. (1999), the effect of homogeneous shear on turbulence is modelled by making the quantity $c_{\mu}$ a function of the dimensionless shear parameter $\eta$. It is the first time that this feature is introduced to multi-scale models. The expression for $c_{\mu}$ proposed here is then:

$$
c_{\mu}=\min \left[c_{\mu_{B L}}, c_{1}+c_{2} \exp \left(-c_{3} \eta\right)\right]
$$

where $c_{1}, c_{2}$ and $c_{3}$ are coefficients chosen to ensure $c_{\mu}$ decreases appropriately at large strain rates, and $c_{\mu_{B L}}$ is the value $c_{\mu}$ is expected to reach in the local equilibrium region of zero-pressure-gradient boundary layers, that is, when $\eta=\frac{k}{\varepsilon_{T}} \frac{d U}{d y} \approx 3.3$ (hence in STS models $c_{\mu_{B L}}=0.09$ ). This clipping has been introduced in order to guarantee stability in regions of very low shear, where a low $\eta$ would otherwise result in $c_{\mu}$ values higher than that at local equilibrium, as also noted and applied in Craft et al. (1999).

Before proceeding with the modelling details of the proposed models, the asymptotic analyses considered here are discussed.

\subsection{Asymptotic Analysis of Homogeneous Shear Flows}

Homogeneous shear flows can be set up by subjecting an isotropic flow to a fixed shear $\left(S=\frac{d U}{d y}\right)$. Since the velocity field is fixed, physical boundaries can be ignored and transport equations can be simplified by neglecting the diffusion terms:

$$
\begin{gathered}
\frac{D k_{P}}{D t}=P_{k}-\varepsilon_{P} \\
\frac{D k_{T}}{D t}=\varepsilon_{P}-\varepsilon_{T} \\
\frac{D \varepsilon_{P}}{D t}=C_{P 1} P_{k} \frac{\varepsilon_{P}}{k_{P}}-C_{P 2} \frac{\varepsilon_{P}^{2}}{k_{P}}+C_{P 1}^{\prime} k_{P}\left(\frac{\partial U}{\partial y}\right)^{2} \\
\frac{D \varepsilon_{T}}{D t}=C_{T 1} \frac{\varepsilon_{P} \varepsilon_{T}}{k_{T}}-C_{T 2} \frac{\varepsilon_{T}^{2}}{k_{T}}
\end{gathered}
$$

The temporal evolution of the turbulence field can be evaluated in an analytical manner when the asymptotic condition is reached, which is identified by constant ratios of turbulent quantities, implying their substantial derivatives become zero: $\frac{D}{D t}\left(\frac{k}{\varepsilon_{T}}\right), \frac{D}{D t}\left(\frac{k_{T}}{\varepsilon_{T}}\right), \frac{D}{D t}\left(\frac{k_{P}}{k_{T}}\right), \frac{D}{D t}\left(\frac{k_{P}}{\varepsilon_{T}}\right), \frac{D}{D t}\left(\frac{k_{P}}{\varepsilon_{P}}\right)$, $\frac{D}{D t}\left(\frac{k_{T}}{\varepsilon_{P}}\right)$ and $\frac{D}{D t}\left(\frac{\varepsilon_{P}}{\varepsilon_{T}}\right)$ all become zero.

In order to carry out the asymptotic analysis not all the above ratios are necessary. The ones which have been employed in this analysis are: $\frac{D}{D t}\left(\frac{k_{T}}{\varepsilon_{T}}\right)=0, \frac{D}{D t}\left(\frac{k}{\varepsilon_{T}}\right)=0$ and $\frac{D}{D t}\left(\frac{k_{P}}{\varepsilon_{P}}\right)=0$, since 
they are enough to allow one to find relations for $\left(\frac{\varepsilon_{P}}{\varepsilon_{T}}\right)_{S F},\left(\frac{k_{P}}{k_{T}}\right)_{S F}$ and $\left(\frac{P_{k}}{\varepsilon_{P}}\right)_{S F}$ respectively, where the subscript $S F$ indicates the value of the ratio in the asymptotic state of homogeneous shear flows. Applying the chain rule for these derivatives and using the substantial derivatives for the partition variables, given in equations (7) to (10), it is possible to derive equations (11) to (13) below:

$$
\begin{gathered}
\left(\frac{\varepsilon_{P}}{\varepsilon_{T}}\right)_{S F}=\left(\frac{1-C_{T 2}}{1-C_{T 1}}\right)_{S F} \\
\left(\frac{k_{P}}{k_{T}}\right)_{S F}=\left(\frac{\frac{P_{k}}{\varepsilon_{T}}-\frac{\varepsilon_{P}}{\varepsilon_{T}}}{\frac{\varepsilon_{P}}{\varepsilon_{T}}-1}\right)_{S F} \\
\left(\frac{P_{k}}{\varepsilon_{P}}\right)_{S F}=\left[\frac{1-C_{P 2}}{1-C_{P 1}-\frac{C_{P 1}^{\prime}}{\nu_{t}} \frac{k_{P}^{2}}{\varepsilon_{P}}}\right]_{S F}
\end{gathered}
$$

DNS studies (Rogers and Moin, 1987; Matsumoto et al., 1991; Lee et al., 1990) suggest that 1.9 is a reasonable value for the asymptotic state of the ratio $\left(\frac{P_{k}}{\varepsilon}\right)_{S F}$, which is also consistent with the values a number of benchmark turbulence model such as the $k-\varepsilon$ and Reynolds stress transport models of Gibson and Launder (1978) and Speziale et al. (1991) predict, so this value has been adopted here for equation (12). Equation (13) shows that $\left(\frac{P_{k}}{\varepsilon_{P}}\right)_{S F}$ depends on the expression used for the eddy viscosity. This will be presented below for the two models developed here, since they use different expressions for $\nu_{t}$.

\subsection{Asymptotic Analysis of Decaying grid Turbulence}

Decaying grid turbulence involves an initially uniform flow, which can be generated downstream of a turbulence generating grid. Due to the absence of strain no production mechanism is present in the flow and consequently only dissipation plays a role, leading to an asymptotic state. Therefore, at this stage, production and diffusion terms can be neglected. Equations (2) to (5) reduce to:

$$
\begin{gathered}
\frac{D k_{P}}{D t}=-\varepsilon_{P} \\
\frac{D k_{T}}{D t}=\varepsilon_{P}-\varepsilon_{T} \\
\frac{D \varepsilon_{P}}{D t}=-C_{P 2} \frac{\varepsilon_{P}^{2}}{k_{P}} \\
\frac{D \varepsilon_{T}}{D t}=C_{T 1} \frac{\varepsilon_{P} \varepsilon_{T}}{k_{T}}-C_{T 2} \frac{\varepsilon_{T}^{2}}{k_{T}}
\end{gathered}
$$

Experiments of decaying grid turbulence show that the evolution (or decay) of $k$ with time is proportional to $t^{-n}$. Hanjalic et al. (1980) adopted the value of $n=1.25$ as a reasonable estimate which is also consistent with the work of Kang et al. (2003) and that is then the value of $n$ assumed here too.

Hanjalic et al. (1980) assumed that $\frac{k_{P}}{k_{T}}$ becomes constant during the decay process which would imply $C_{P 2}=\frac{n+1}{n}=1.8$ (with $n=1.25$ ), as normally found in STS models. Consequently $\frac{\varepsilon_{P}}{\varepsilon_{T}}$ can also be considered constant in the asymptotic state which is equivalent to $\frac{D}{D t}\left(\frac{k_{P}}{k_{T}}\right)=0$ and $\frac{D}{D t}\left(\frac{\varepsilon_{P}}{\varepsilon_{T}}\right)=0$. The application of these conditions in equations (2) to (5) results in the following relations (where the subscript $D F$ denotes asymptotic state of decaying grid turbulence) for these spectral ratios as functions of of the model's coefficients:

$$
\left(\frac{\varepsilon_{P}}{\varepsilon_{T}}\right)_{D F}=\left(\frac{C_{T 2}-C_{P 2}}{C_{T 1}-C_{P 2}}\right)_{D F}
$$




$$
\left(\frac{k_{P}}{k_{T}}\right)_{D F}=\left(\frac{C_{P 2}-C_{T 2}}{C_{T 2}-C_{T 1}}\right)_{D F}=\frac{\frac{\varepsilon_{P}}{\varepsilon_{T}}}{1-\frac{\varepsilon_{P}}{\varepsilon_{T}}}
$$

It is worth commenting that Hanjalic et al. (1980) imposed $\left(\frac{k_{P}}{k_{T}}\right)_{D F}=1$. This assumption was not adopted here, since no evidence was found to support this. It must be expected that $\frac{k_{P}}{k_{T}}$ is lowest in decaying grid turbulence, $\left(\frac{k_{P}}{k_{T}}\right)_{D F}$ representing the minimum ratio one should expect to find in any flow, since no production is present. Thereby this ratio becomes a useful parameter with which to check the consistency of the model in different flows and can also be used as a limit to adjust coefficients in the modelling process.

\subsection{Asymptotic Analysis of Local Equilibrium Boundary Layers}

The classical theory of zero-pressure-gradient boundary layers, in which turbulence is in local equilibrium, has also been applied to two-time-scale models. In such flows, convection can be neglected and the generation rate of turbulence equals the eddy dissipation rate, that is, $P_{k}=$ $\varepsilon=\varepsilon_{T}$. Moreover, if equilibrium is considered, the rate at which energy is transferred from the production to the transfer zone equals the rate at which energy is transferred from the transfer to the dissipation zone, that is, $\varepsilon_{P}=\varepsilon_{T}$. If the subscript $B L$ is used to denote the asymptotic (or equilibrium) state of local equilibrium boundary layers, then one can write $\left(\frac{\varepsilon_{P}}{\varepsilon_{T}}\right)_{B L}=1$.

The assumptions of a logarithmic velocity variation and a constant shear stress across the boundary layer yield relations such as $\frac{d U}{d y}=\frac{u_{\tau}}{\kappa y},-\overline{u v}=\frac{\tau_{w}}{\rho}=u_{\tau}^{2}, P_{k}=\frac{u_{\tau}^{3}}{\kappa y}$ and $\nu_{t}=u_{\tau} \kappa y$ which remain valid when considering two-time-scale models. These relations can be thus substituted into equations (4) and (5), applied to a 2-D boundary layer, where variations occur mainly in the direction perpendicular to the flow, and their left hand sides can be set as zero, since convection is neglected:

$$
\begin{gathered}
0=\frac{\left(u_{\tau}^{3} / \kappa y\right)^{2}}{k_{P}}\left(C_{P 1}-C_{P 2}\right)+\frac{\partial}{\partial y}\left[\frac{\nu_{t}}{\sigma_{\varepsilon_{P}}} \frac{\partial\left(u_{\tau}^{3} / \kappa y\right)}{\partial y}\right]+C_{P 1}^{\prime} k_{P}\left(\frac{\partial U}{\partial y}\right)^{2} \\
0=\frac{\left(u_{\tau}^{3} / \kappa y\right)^{2}}{k_{T}}\left(C_{T 1}-C_{T 2}\right)+\frac{\partial}{\partial y}\left[\frac{\nu_{t}}{\sigma_{\varepsilon_{T}}} \frac{\partial\left(u_{\tau}^{3} / \kappa y\right)}{\partial y}\right]
\end{gathered}
$$

Further development of the above equations depends on the eddy viscosity formulation used and leads to a relation between the coefficients of the model as well as the the asymptotic value expected for the ratio $\left(\frac{k_{P}}{k_{T}}\right)_{B L}$. Therefore, these results are presented separately for each of the models developed below.

It is worth commenting here that tests carried out indicated that in order to respect spectral relations which are consistent with the turbulent kinetic energy spectrum and the cascade process, such as the ratio between the small and large time and length scales $\left(\tau_{T}=\frac{k_{T}}{\varepsilon_{T}}, \tau_{P}=\frac{k_{P}}{\varepsilon_{P}}, \ell_{T}=\frac{k_{T}^{3 / 2}}{\varepsilon_{T}}\right.$ and $\ell_{P}=\frac{k_{P}^{3 / 2}}{\varepsilon_{P}}$ respectively) being always smaller than unity $\left(\frac{\tau_{T}}{\tau_{P}}<1\right.$ and $\left.\frac{\ell_{T}}{\ell_{P}}<1\right)$ the ratio $\left(\frac{k_{P}}{k_{T}}\right)_{B L}$ should be around, or higher than, 3. Indeed, Stawiarski and Hanjalic (2005) also support that 3 would be a reasonable value for the ratio $\left(\frac{k_{P}}{k_{T}}\right)_{B L}$ and mentioned that experiments suggest that this ratio could be even higher than 3 . Therefore this is another parameter that arises and can be used as a modelling tool for coefficients in a model.

\subsection{The NT1 Model}

The first model developed here, the so-called NT1 model, is defined as using the general equations (2) to (5) presented above and keeping the expression for the eddy viscosity as proposed by Hanjalic et al. (1980): 


$$
\nu_{t}=c_{\mu} \frac{k k_{P}}{\varepsilon_{P}}
$$

For the NT1 formulation, simulations of homogeneous shear flows carried out during the course of this investigation show that the last term in equation (4) is a source of instability in high shear flows and therefore such a term should either be removed or associated with a much lower coefficient. In the present formulation, it has been found that removing this term is indeed the best option. So, for the NT1 model, the coefficient $C_{P 1}^{\prime}$ is set to zero.

Initially, the general form of the coefficients proposed by Hanjalic et al. (1980) was retained. That implied taking $C_{P 1}, C_{T 2}, C_{P 1}^{\prime}, \sigma_{k_{P}}, \sigma_{k_{T}}, \sigma_{\varepsilon_{P}}$ and $\sigma_{\varepsilon_{T}}$ as constants and using the following form for the two remaining coefficients: $C_{P 2}=c_{p 21}-c_{p 22}\left(\frac{k_{P}}{k_{T}}-1\right) /\left(\frac{k_{P}}{k_{T}}+1\right)$ and $C_{T 1}=c_{t 1} \frac{\varepsilon_{P}}{\varepsilon_{T}}$, where $c_{p 21}, c_{p 22}$ and $c_{t 1}$ are also constants. This expression proposed by Hanjalic et al. (1980) makes the coefficient $C_{P 2}$ to decrease smoothly as $\frac{k_{P}}{k_{T}}$ increases and to tend to a constant value as $\frac{k_{P}}{k_{T}}$ becomes large.

In the case of homogeneous flows subjected to low shear, at the start of the temporal evolution of the turbulence field, the ratio $\frac{k_{P}}{k_{T}}$ is reduced. In order for the models to reproduce this, the source term in the $k_{P}$ equation needed to be reduced. A reduction of the $k_{P}$ source term, in such flow conditions, can be achieved by sensitising $C_{P 1}$ to the ratio $\frac{k_{P}}{k_{T}}$. The following form is then adopted, in analogy to the expression proposed by Hanjalic et al. (1980) to $C_{P 2}$, in order to provide improvements and at the same time not affect the correct prediction of the asymptotic case of local equilibrium boundary layers:

$$
C_{P 1}=c_{p 11}+c_{p 12} \min \left[0, \frac{k_{P}}{k_{T}}-\left(\frac{k_{P}}{k_{T}}\right)_{B L}\right] /\left[\frac{k_{P}}{k_{T}}+\left(\frac{k_{P}}{k_{T}}\right)_{B L}\right]
$$

with $c_{p 11}$ and $c_{p 12}$ being constants. For values of $\frac{k_{P}}{k_{T}}>\left(\frac{k_{P}}{k_{T}}\right)_{B L}$, the coefficient $C_{P 1}$ reduces to $C_{P 1}=c_{p 11}$. Tests have also been carried out considering $C_{T 1}$ as constant, that is, making $C_{T 1}=c_{t 1}$. All these constants have been adjusted in order to provide the best prediction of homogeneous shear flows in the range of dimensionless shear considered.

With the eddy viscosity given by equation $(22)$ and $C_{P 1}^{\prime}=0$, the asymptotic analysis for homogeneous shear flows and local equilibrium boundary layers results in the following relations:

$$
\begin{gathered}
\left(\frac{P_{k}}{\varepsilon_{T}}\right)_{S F}=\left(\frac{P_{k}}{\varepsilon_{P}}\right)_{S F}\left(\frac{\varepsilon_{P}}{\varepsilon_{T}}\right)_{S F}=1.9=\left[\frac{1-C_{P 2}}{1-C_{P 1}} \frac{1-C_{T 2}}{1-C_{T 1}}\right]_{S F} \\
\left(\frac{k_{P}}{k_{T}}\right)_{B L}=\left[\frac{\sigma_{\varepsilon_{P}}}{\sigma_{\varepsilon_{T}}} \frac{\left(C_{P 2}-C_{P 1}\right)}{\left(C_{T 2}-C_{T 1}\right)}\right]_{B L} \\
\left\{\left(C_{P 2}-C_{P 1}\right)\left[\sigma_{\varepsilon_{P}}\left(C_{P 2}-C_{P 1}\right)+\sigma_{\varepsilon_{T}}\left(C_{T 2}-C_{T 1}\right)\right]\right\}_{B L}=\left(\frac{\kappa^{4}}{c_{\mu} \sigma_{\varepsilon_{P}}}\right)_{B L} \\
c_{\mu}\left(\frac{k_{P}}{k}\right)_{B L} \approx 0.09
\end{gathered}
$$

Since $k_{P} / k$ is necessarily smaller than one, $c_{\mu}$ will necessarily assume a value higher than 0.09 which is its traditional value used in single-time-scale models.

Equations (11), (12), (24) (from homogeneous shear flows), (18), (19) (from decaying grid turbulence) and (25) to (27) (from local equilibrium boundary layers) comprise the set of constraint equations for the NT1 model which ensure correct prediction for these three cases. It is of value to note that, according to the asymptotic analysis of decaying grid turbulence, based on equations (14) to (17), although $C_{P 2}$ is not constant, in this asymptotic state it should be around 1.8. Therefore, $C_{P 2_{D F}}$, which is the value of the coefficient $C_{P 2}$ in the asymptotic state of decaying grid turbulence, should be 1.8. Equally, in all the above mentioned equations, whenever a coefficient is dependent on the spectral ratios $\frac{k_{P}}{k_{T}}$ and/or $\frac{\varepsilon_{P}}{\varepsilon_{T}}$, their values should be taken accordingly. For 
example, for asymptotic conditions of homogeneous shear flows, $C_{P 2}$ should assume the following form in equation (24):

$$
C_{P 2}=c_{p 21}-c_{p 22}\left[\left(k_{P} / k_{T}\right)_{S F}-1\right] /\left[\left(k_{P} / k_{T}\right)_{S F}+1\right]
$$

For asymptotic conditions of local equilibrium boundary layers, $C_{P 2}$ should assume the following form in equation (25):

$$
C_{P 2}=c_{p 21}-c_{p 22}\left[\left(k_{P} / k_{T}\right)_{B L}-1\right] /\left[\left(k_{P} / k_{T}\right)_{B L}+1\right]
$$

The set of coefficients which satisfy the set of constraint equations and provide good predictions for a wide range of dimensionless shear cases in homogeneous flows is presented in Table 1. The coefficients $\sigma_{k_{P}}$ and $\sigma_{k_{T}}$ do not appear in any of the constraint equations and tests performed by the authors indicated the values of unity (similarly to single-time-scale models) would fit other cases best. These coefficients complete the NT1 model which then provides the asymptotic spectral ratios presented in Table 3 for further comparison with the NT2 model (described below).

Table 1: Coefficients of the NT1 model

\begin{tabular}{ccccccc}
\hline \hline$c_{\mu}$ & $C_{P 1}$ & $C_{P 2}$ & $\sigma_{\varepsilon_{P}}$ & $C_{T 1}$ & $C_{T 2}$ & $\sigma_{\varepsilon_{T}}$ \\
\hline $\min \left[0.115,0.023+0.25 e^{-0.30 \eta}\right]$ & $\max \left[1.4912+2.5 \frac{\min \left(0, \frac{k_{P}}{k_{T}}-f_{B L}\right)}{\frac{k_{P}}{k_{T}}+f_{B L}}, 0\right]$ & 1.8 & 1.4202 & 1.6 & 1.7 & 1.2181 \\
\hline
\end{tabular}

Commenting on Table 1, it should be pointed out that the only non-constant coefficient is $C_{P 1}$ where it may be noted that an extra term $\left(f_{B L}\right)$ has been inserted. This term is defined by the equations below:

$$
\begin{gathered}
f_{B L}=\left(\frac{k_{P}}{k_{T}}\right)_{B L}\left(1-f_{1}+f_{2}\right)=3.6\left(1-f_{1}+f_{2}\right) \\
f_{1}=1.7\left(\frac{k_{P}}{k_{T}}-1\right) \min \left(\frac{P_{k}}{\varepsilon_{T}}-1.9,0\right) \max \left(\frac{\varepsilon_{P}}{\varepsilon_{T}}-1,0\right) \\
f_{2}=20 \max \left[\frac{k_{P}}{k_{T}} \max \left(\frac{\varepsilon_{P}}{\varepsilon_{T}}-1.1,0\right) S W, 0\right] \\
S W=\frac{S_{i j} S_{i j}-W_{i j} W_{i j}}{S_{i j} S_{i j}+W_{i j} W_{i j}}
\end{gathered}
$$

The reason why $f_{B L}$ has been inserted into the $C_{P 1}$ coefficient of the NT1 model is to improve prediction in strongly non-equilibrium flows such as backward facing step flows. During the current study it was established that models optimized to satisfy asymptotic states of simple flows are not able to predict the effects present in strongly non-equilibrium shear regions. The $f_{B L}$ term has then been designed to improve sensitivity in these non-equilibrium shear regions and at the same time not affect any of the asymptotic analyses previously discussed, when it reduces to $\left(\frac{k_{P}}{k_{T}}\right)_{B L}=3.6$.

As will be shown in section 3.8, the HLS model strongly underpredicts the reattachment length in steady state backward facing step cases. The NT1 model without the $f_{B L}$ term (equations (30) to (33)) tended to overpredict this parameter. Hence, the $f_{B L}$ term has been designed to decrease the reattachment length predicted by the NT1 model. It was arrived at by considering the profiles of $\frac{k_{P}}{k_{T}}$ and $\frac{\varepsilon_{P}}{\varepsilon_{T}}$ predicted by both HLS and NT1 models in backward facing step cases. No significant differences were noted in the $\frac{\varepsilon_{P}}{\varepsilon_{T}}$ profiles, but the HLS model provided much higher levels of $\frac{k_{P}}{k_{T}}$ than the NT1 model in the shear regions of the flow. The $f_{B L}$ function has thus been designed to increase levels of $\frac{k_{P}}{k_{T}}$ in such strongly sheared regions, through its influence on the $C_{P 1}$ coefficient, which is associated with the production term in the $k_{P}$ equation. The function $f_{1}$ is built up from three parts. The term $\max \left(\varepsilon_{P} / \varepsilon_{T}-1,0\right)$ is the one mainly responsible for improving prediction of 
backward facing step cases, since it had been noticed that $\varepsilon_{P} / \varepsilon_{T}$ assumes values higher than 1.0 in strongly sheared regions, 1.0 being the equilibrium value for this spectral ratio in local equilibrium boundary layers. The term $\min \left(P_{k} / \varepsilon_{T}-1.9,0\right)$ ensures that the function does not significantly affect the homogeneous shear flow predictions, where $P_{k} / \varepsilon_{T}=1.9$ is the expected asymptotic value and where, during the temporal evolution of the turbulence field, $\varepsilon_{P} / \varepsilon_{T}$ can assume values considerably higher than 1.0, although its asymptotic value is just slightly higher than unity. Finally, the term $\left(k_{P} / k_{T}-1\right)$ has been introduced into $f_{1}$ in order to reduce any unexpected influence of this function in regions of low turbulence levels where $k_{P} / k_{T}$ may approach its minimum predicted value of 1.0, which is the asymptotic value for this spectral ratio in decaying grid turbulence flows. As a side effect, this term also increased the overall value of $f_{1}$ whose coefficient was thus set to a relatively small value of 1.7. It can also be noted that the terms $\max \left(\varepsilon_{P} / \varepsilon_{T}-1,0\right)$ and $\left(k_{P} / k_{T}-1\right)$ are always positive and the term $\min \left(P_{k} / \varepsilon_{T}-1.9,0\right)$ is either zero or negative. The minus sign associated with $f_{1}$ thus ensures it acts to increase $f_{B L}$.

Including only the $f_{1}$ term in $f_{B L}$ significantly improved the prediction of the reattachment length in both steady state backward facing step and in the case where a high frequency (Strouhal number $S t=1$ ) control jet is introduced at the step corner. However, in lower frequency forced cases the reattachment length was still not reduced enough. It was then identified that the term $S W$, defined in equation (33), came out as nearly zero in both steady state and high frequency $(S t=1)$ backward facing step cases, but non-zero in the low frequency backward facing step case $(S t=0.275)$. Moreover, $S W$ is zero in simple shear flows, such as homogeneous shear and boundary layer flows. Therefore, the $f_{2}$ function, expressed in equation (32), has been developed, taking into account that the term $\max \left(\varepsilon_{P} / \varepsilon_{T}-1.1,0\right)$ is non-zero only when $\varepsilon_{P} / \varepsilon_{T}$ becomes $10 \%$ higher than its equilibrium value in boundary layers, which happens mainly in the low frequency cases when the reattachment point tends to a minimum.

From the above it can be seen that the functions $f_{1}$ and $f_{2}$ are based on the expected asymptotic values of the spectral ratios $k_{P} / k_{T}$ and $\varepsilon_{P} / \varepsilon_{T}$ in homogeneous shear, equilibrium boundary layers and decaying grid turbulence flows.

More details on how expressions in equations (30) to (33) were derived can be found in Klein (2012).

\subsection{The NT2 Model}

The so-called NT2 model has also been defined using the general equations (2) to (5), but now with the expression for the eddy viscosity usually adopted in single-time-scale models, since, according to the turbulent kinetic energy spectrum modelling framework, $\varepsilon_{T}=\varepsilon$ :

$$
\nu_{t}=c_{\mu} \frac{k^{2}}{\varepsilon_{T}}
$$

where the expression for $c_{\mu}$ has been kept as in equation (6).

Computations of homogeneous shear flows established that the extra term proposed by Hanjalic et al. (1980) for the $\varepsilon_{P}$ equation should not now be left out. In order to avoid stability problems, however, its coefficient $C_{P 1}^{\prime}$ has been set to less than a tenth of the value originally proposed by Hanjalic et al. (1980).

The general form for the coefficients in equations (2) to (5) has been kept as described above for the NT1 model and a non-zero value for $C_{P 1}^{\prime}$ was also considered, resulting in the following relations for the NT2 model in the asymptotic analysis of homogeneous shear and local equilibrium boundary layers:

$$
\left(\frac{P_{k}}{\varepsilon_{T}}\right)_{S F}=\left(\frac{P_{k}}{\varepsilon_{P}}\right)_{S F}\left(\frac{\varepsilon_{P}}{\varepsilon_{T}}\right)_{S F}=1.9=\left[\frac{1-C_{P 2}}{1-C_{P 1}-\frac{C_{P 1}^{\prime}}{c_{\mu}}\left(\frac{k_{P}}{k}\right)^{2}\left(\frac{\varepsilon_{T}}{\varepsilon_{P}}\right)}\right]_{S F}\left(\frac{\varepsilon_{P}}{\varepsilon_{T}}\right)_{S F}
$$




$$
\begin{gathered}
\left(\frac{k_{P}}{k_{T}}\right)_{B L}=\frac{\sigma_{\varepsilon_{P}}}{\sigma_{\varepsilon_{T}}}\left[\frac{C_{P 2}-C_{P 1}-\frac{C_{P 1}^{\prime}}{c_{\mu}}\left(\frac{k_{P}}{k}\right)^{2}}{C_{T 2}-C_{T 1}}\right]_{B L} \\
\sigma_{\varepsilon_{P}}\left[C_{P 2}-C_{P 1}-\frac{C_{P 1}^{\prime}}{c_{\mu}}\left(\frac{k_{P}}{k}\right)^{2}\right]_{B L}+\sigma_{\varepsilon_{T}}\left(C_{T 2}-C_{T 1}\right)_{B L}=\frac{\kappa^{2}}{c_{\mu}^{1 / 2}}
\end{gathered}
$$

The set of constraint equations for the NT2 model is then comprised of equations (11), (12), (35) (from homogeneous shear flows), (18), (19) (from decaying grid turbulence), (36) and (37) (from local equilibrium boundary layers). Again, it should be stressed that if $C_{P 2}$ is not constant, its value in the asymptotic state of decaying grid turbulence should be 1.8 . With regard to the asymptotic states of $c_{\mu}$ it is worth recalling that $c_{\mu_{B L}}=0.09$, since the expression for the eddy viscosity is the same as that used for single-time-scale models. However, as $c_{\mu}$ is a function of the dimensionless shear $\eta$ (equation (6)), its asymptotic value in homogeneous shear flows $\left(c_{\mu_{S F}}\right)$ must be known to be used in equation (35). $c_{\mu_{S F}}$ can be obtained by estimating $\eta_{S F}$ through equation (38) below:

$$
\eta_{S F}=\left(\frac{k}{\varepsilon_{T}} \frac{d U}{d y}\right)_{S F}=\left(c_{\mu_{S F}}\right)^{-1 / 2}\left(\frac{P_{k}}{\varepsilon_{T}}\right)_{S F}^{1 / 2}\left(\frac{k}{k_{P}}\right)_{S F}\left(\frac{\varepsilon_{P}}{\varepsilon_{T}}\right)_{S F}^{1 / 2}
$$

but it must be determined iteratively, since $\left(P_{k} / \varepsilon_{T}\right)_{S F},\left(k / k_{P}\right)_{S F}$ and $\left(\varepsilon_{P} / \varepsilon_{T}\right)_{S F}$ also depend on equations (11), (12), (35).

The set of coefficients which satisfies the constraint equations and returns good predictions of homogeneous shear flows is presented in Table 2, thus completing the NT2 model. The asymptotic states of the spectral ratios related to these values are presented in Table 3, together with those provided by the NT1 model for further comparison.

Table 2: Coefficients of the NT2 model

\begin{tabular}{cccc}
\hline \hline$c_{\mu}$ & $C_{T 1}$ & $C_{T 2}$ & $\sigma_{\varepsilon_{T}}$ \\
\hline $\min \left[0.09,0.0117+0.22 e^{-0.31 \eta}\right]$ & $1.0 \frac{\varepsilon_{P}}{\varepsilon_{T}}$ & 1.1 & 1.1922 \\
\hline$C_{P 1}$ & $C_{P 2}$ & $\sigma_{\varepsilon_{P}}$ & $C_{P 1}^{\prime}$ \\
\hline $\max \left[1.5697+2.5 \frac{\min \left(0, \frac{k_{P}}{k_{T}}-f_{B L}\right)}{\frac{k_{P}}{k_{T}}+f_{B L}}, 0\right]$ & 1.8 & 1.6664 & -0.005 \\
\hline
\end{tabular}

Commenting on Table 2, it can be noted that $C_{P 1}$ is not constant and that values for $\sigma_{k_{P}}$ and $\sigma_{k_{T}}$ have not been assigned, because they have both been set to unity in analogy to single-time-scale models, since these parameters do not take part in the constraint equations. The coefficient $C_{T 1}$ in equation (5) can be replaced by its expression presented in Table 2 and the $\varepsilon_{T}$ equation of the NT2 model can be re-written as in equation (39) below, with $c_{t 1}$ being a constant with the value of 1.0:

$$
\frac{D \varepsilon_{T}}{D t}=c_{t 1} \frac{\varepsilon_{P}^{2}}{k_{T}}-C_{T 2} \frac{\varepsilon_{T}^{2}}{k_{T}}+\frac{\partial}{\partial x_{j}}\left[\left(\nu+\frac{\nu_{t}}{\sigma_{\varepsilon_{T}}}\right) \frac{\partial \varepsilon_{T}}{\partial x_{j}}\right]
$$

As shown in Table 2, an extra term also had to be inserted into the NT2 model expression for the coefficient $C_{P 1}$ in order to improve prediction of strongly non-equilibrium shear regions such as backward facing step flows. This extra term, $f_{B L}$, is defined by equations (40) to (42) and does not affect the asymptotic analyses carried out before, since in these situations it reduces to the value of $\left(\frac{k_{P}}{k_{T}}\right)_{B L}=3.7$. The procedure to arrive at these expressions is similar to that described above for the NT1 model. It is informative to highlight that the value of 1.3166, which appears in $f_{1}$ in equation (41), is the minimum expected value for this ratio, since it is the asymptotic value for $k_{P} / k_{T}$ in decaying grid flows. 


$$
\begin{gathered}
f_{B L}=\left(\frac{k_{P}}{k_{T}}\right)_{B L}\left(1-f_{1}+f_{2}\right)=3.7\left(1-f_{1}+f_{2}\right) \\
f_{1}=5.4\left(\frac{k_{P}}{k_{T}}-1.3166\right) \min \left(\frac{P_{k}}{\varepsilon_{T}}-1.9,0\right) \max \left(\frac{\varepsilon_{P}}{\varepsilon_{T}}-1,0\right) \\
f_{2}=11 \min \left\{1.8, \max \left[\frac{k_{P}}{k_{T}} \max \left(\frac{\varepsilon_{P}}{\varepsilon_{T}}-1,0\right) S W, 0\right]\right\}
\end{gathered}
$$

It can be noted in both Tables 1 and 2 that the coefficient $C_{P 1}$ has been clipped in order to be always positive, as expected for the source term of the $\varepsilon$ equation in linear-eddy-viscosity models.

\subsection{Comparison of NT1 and NT2 Models}

In Table 3 the values of several model parameters expected to be predicted by the NT1 and NT2 models in asymptotic conditions are presented together for comparison. It should be noted that although $\left(\frac{P_{k}}{\varepsilon_{T}}\right)_{S F}$ is included, this value is specified before the asymptotic analyses. The expected values for the ratio $\frac{k_{P}}{k_{T}}$ are higher for the NT2 model than for NT1, specially in homogeneous shear flows. That means the NT1 and NT2 models split the spectrum at quite different positions. Both models, however, can be shown (Klein, 2012) to place this split within the region where viscous effects are negligible, but where the length, velocity and time scales are expected to be lower than those of the main flow, consistent with the inertial subrange of Kolmogorov's theory for the turbulent kinetic energy spectrum. More important though is to notice that for the NT1 and NT2 models $\left(\frac{k_{P}}{k_{T}}\right)_{D F}<\left(\frac{k_{P}}{k_{T}}\right)_{B L}<\left(\frac{k_{P}}{k_{T}}\right)_{S F}$ which is expected, given the levels of turbulence for each asymptotic flow. The ratios of the dissipation rates of the two zones $\left(\frac{\varepsilon_{P}}{\varepsilon_{T}}\right)_{D F}$ predicted by both NT1 and NT2 models are also consistent with equation (19), since they are less than one in order to lead to a positive ratio $\left(\frac{k_{P}}{k_{T}}\right)_{D F}$. Also worth noting is that, although not pre-specified, as was done in Hanjalic et al. (1980), in NT1 the ratio $\left(\frac{k_{P}}{k_{T}}\right)_{D F}$ is predicted to have the value of 1 . It must also be emphasized that the parameters used for clipping in the expressions of the extra terms inserted into the coefficient $C_{P 1}$ - equations (31), (32), (41) and (42) - are related to the asymptotic states expected to be predicted by the NT1 and NT2 models.

Table 3: Values to be provided by the NT1 and NT2 models in the asymptotic states studied

\begin{tabular}{cccccccccc}
\hline \hline & $\left(\frac{P_{k}}{\varepsilon_{T}}\right)_{S F}$ & $\left(\frac{k_{P}}{k_{T}}\right)_{S F}$ & $\left(\frac{\varepsilon_{P}}{\varepsilon_{T}}\right)_{S F}$ & $\left(\frac{k_{P}}{k_{T}}\right)_{D F}$ & $\left(\frac{\varepsilon_{P}}{\varepsilon_{T}}\right)_{D F}$ & $\left(\frac{k_{P}}{k_{T}}\right)_{B L}$ & $\left(\frac{\varepsilon_{P}}{\varepsilon_{T}}\right)_{B L}$ & $c_{\mu_{B L}}$ & $c_{\mu_{S F}}$ \\
\hline NT1 & 1.9 & 4.4000 & 1.1667 & 1.0000 & 0.5000 & 3.6 & 1.0 & 0.115 & 0.0338 \\
\hline NT2 & 1.9 & 8.8245 & 1.0916 & 1.3166 & 0.5683 & 3.7 & 1.0 & 0.090 & 0.0336 \\
\hline
\end{tabular}

\section{Performance of the Turbulence Models}

In order to assess the performance of the two two-time-scale linear-eddy-viscosity models NT1 and NT2, their performance is compared to those of the two-time-scale linear-eddy-viscosity model of Hanjalic et al. (1980), which has been the starting point for further model developments in this work, of the standard $k-\varepsilon$ model which is a single-time-scale linear-eddy-viscosity model, and of the single-time-scale Reynolds-stress-transport model of Speziale et al. (1991). The performance of other turbulence models for the same test cases considered here can be found in Klein et al. (2015). In the results, the model of Hanjalic et al. (1980) will be referred to as the HLS model and that of Speziale et al. (1991) as the SSG model.

All the models considered here are high-Reynolds-number approaches, which means they must be used with a wall function. The wall function used here is the log-law based formulation proposed 


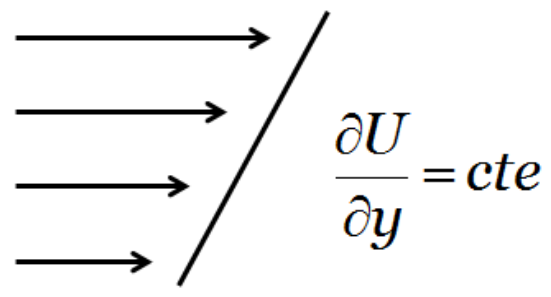

Figure 1: Homogeneous shear flow diagram

by Chieng and Launder (1980). In the case of two-time-scale models, the wall function is applied to the equations of $k_{P}$ and $\varepsilon_{T}$ and a relation between $\frac{k_{P}}{k_{T}}$ and $\frac{\varepsilon_{P}}{\varepsilon_{T}}$ is specified to evaluate the $k_{T}$ and $\varepsilon_{P}$ at the near-wall nodes. In accord with the local equilibrium requirement, which is also employed in this wall-function strategy, the ratio $\frac{\varepsilon_{P}}{\varepsilon_{T}}$ is set to unity. For the NT1 and NT2 models, the value of the ratio between $k_{P}$ and $k_{T}$ is taken from Table 3 as the value of $\left(\frac{k_{P}}{k_{T}}\right)_{B L}$ predicted by each model. For the HLS model, this ratio is set to 2, as discussed in Klein et al. (2015).

Tests have first been carried out for simple flows such as channel flows and zero-pressuregradient boundary layers which demonstrated that the models perform reasonably well in such simple equilibrium flows. Comparisons for these basic cases have not been included, to avoid making this communication unnecessarily long. The performance of the models is then assessed over a wide range of non-equilibrium flows:

- Homogeneous shear flows

- Normally strained flows

- Adverse-pressure-gradient boundary layer flows

- Favourable-pressure-gradient boundary layer flows

- Oscillatory boundary layer flows

- Fully developed oscillatory pipe flows

- Fully developed ramp up pipe flows

- Steady and pulsated backward-facing-step flows

All test cases have been simulated using the in-house CFD code STREAM, described in Lien and Leschziner (1994). This is a finite-volume CFD code, which employs a fully-collocated grid arrangement and uses the SIMPLE pressure correction algorithm, with the Rhie and Chow (1983) flux modification. In all the cases reported below, checks have been taken to ensure the quoted grid resolutions and time-steps were sufficient for grid and time-step independence to be achieved. More details on the turbulence models as well as on each test case can be found in Klein (2012) or Klein et al. (2015).

Here only the prediction of the turbulent quantities such as the Reynolds shear stress, turbulent kinetic energy and eddy dissipation rate are assessed, since as linear-eddy-viscosity models, the NT1 and NT2 models are not expected to provide accurate prediction of the Reynolds normal stresses.

\subsection{Homogeneous Shear Flows}

As discussed before, in homogeneous shear flows, the flow field is fixed, as shown in Figure 1, and the simulations involve the solution of the one-dimensional time-dependent transport equations for the turbulence parameters, which provide the temporal evolution of the turbulence field.

Some homogeneous shear flows have been used for the tuning of the NT1 and NT2 models' coefficients, as discussed in sections 2.4 and 2.5. The cases discussed here involve some cases which 
have been used in the tuning process, but also additional homogeneous shear flow cases, which did not form part of the tuning process.

A low shear case from Rogers and Moin (1987) has been selected to present here, with initial dimensionless shear values of $\eta_{0}=\frac{k_{0}}{\varepsilon_{0}} S=1.2(S=d U / d y)$. A moderate shear case has then been selected from Matsumoto et al. (1991) with $\eta_{0}=4.7$ and a high shear case from Lee et al. (1990) with $\eta_{0}=16.8$, thereby covering a wide range of shear intensity. The flow properties and initial conditions of each case have been taken as reported in their original papers and a timestep of $\Delta t=\frac{1}{10 S}$ has been used.

The presence of a homogeneous shear leads to generation of turbulence throughout the flow domain. Consequently, as a fluid with low isotropic turbulence is subjected to homogeneous shear, its turbulent kinetic energy will start to increase. This increase in turbulence will then further strengthen the shear generation rate of turbulence. As a result, it is expected that the turbulent kinetic energy will rise exponentially in time and that this exponential rate of increase will become stronger at higher dimensionless shear rates. This is what is shown by the DNS in Figure 2. The DNS data also show that the dissipation rate increases in the same manner as the turbulent kinetic energy, since higher turbulence levels also lead to higher rates of transfer of energy to the smaller length scales. The turbulent shear stress also rises in magnitude as the flow develops.

As discussed in Klein et al. (2015), most turbulence models, while qualitatively following these trends, they over predict the levels of the turbulent quantities at moderate and high shear levels. It is evident from Figure 2 though, that the introduction of the proposed models NT1 and NT2 results in clear improvements in the predictions of the turbulent kinetic energy, the eddy dissipation rate and the Reynolds shear stress, over the entire range of dimensionless stress levels tested. In the low shear case, all five models return reasonable predictions, although the NT1 and NT2 models are clearly closer to the DNS data. In the moderate and high shear cases only the NT1 and NT2 models are able to remain close to the DNS data, thus providing the only reliable set of predictions.

The main feature in the models proposed here which provides such improvements in predictions is the non-constant $c_{\mu}$, which here depends on the dimensionless shear parameter $\eta$, as shown in equation (6). It is important to highlight though that the multi-scale approach also contributes to the improvements in performance. Additional tests have been carried out with the standard $k-\varepsilon$ model, using $c_{\mu}$ as a function of $\eta$ and, although improvements have been found, the predictions are not as close to the DNS data as those of the NT1 and NT2 models.

The slight kink in the profile of the Reynolds shear stress provided by the NT1 and NT2 models in the low shear case at around $S t=2$, seen in Figure 2(c), is due to the upper bound in $c_{\mu}$ which has been introduced to avoid numerical stability problems in regions where the mean velocity undergoes strong changes, as commented on earlier.

\subsection{Normally Strained Flows}

Normally strained flows are those subjected to homogeneous normal straining, as shown in Figure 3 , and therefore the Reynolds normal stresses are expected to play an important role. In spite of this, the prediction of the evolution of the turbulent kinetic energy can still be assessed by linear-eddy-viscosity models.

In Figure 4 the evolution in time of $k$ is shown and compared for two different cases from experimental investigations: the laterally distorting tunnel of Tucker and Reynolds (1968) with normal straining of $\frac{d W}{d z}=-\frac{d V}{d y}=4.45 \mathrm{~s}^{-1}$ and inlet velocity of $U=6.1 \mathrm{~m} / \mathrm{s}$; and the pure plane strain case of Gence and Mathieu (1979) with normal straining of $\frac{d W}{d z}=-\frac{d V}{d y}=32.23 \mathrm{~s}^{-1}$ and inlet velocity of $U=18.6 \mathrm{~m} / \mathrm{s}$. While the latter case applied the normal straining along the entire length of the $0.8 \mathrm{~m}$ long duct, Tucker and Reynolds (1968) let a quasi-isotropic turbulent flow develop for $0.61 \mathrm{~m}$, then applied a constant symmetric plane strain for $2.44 \mathrm{~m}$ and finally let the flow recover for $1.22 \mathrm{~m}$ more. A dimensionless straining history can be defined as $\eta=\frac{k}{\varepsilon}\left|\frac{d V}{d y}\right|$ and plotted against a dimensionless streamwise distance defined as $\frac{x}{U}\left|\frac{d V}{d y}\right|$ in order to characterize each of the cases simulated. It can thus be said that the the laterally distorting tunnel of Tucker and Reynolds 


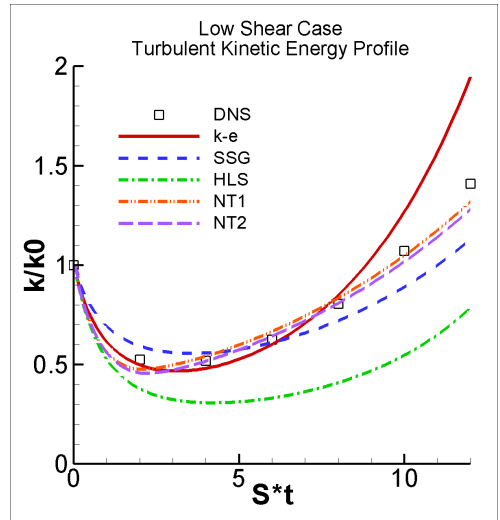

(a)

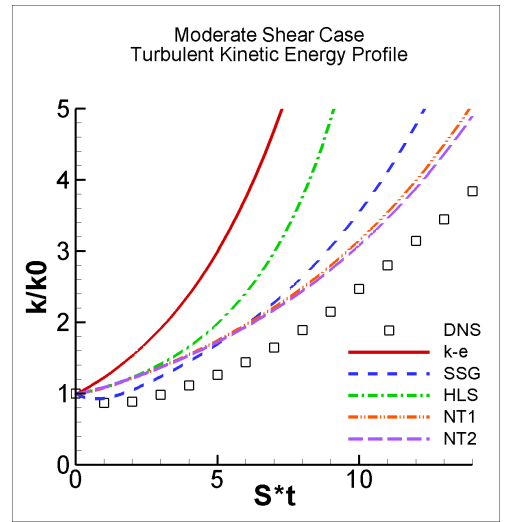

(d)

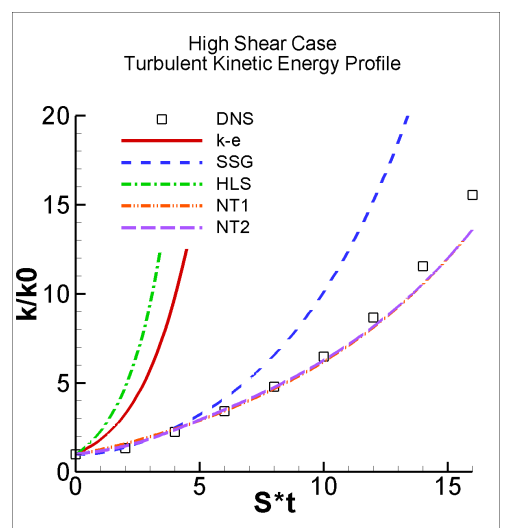

(g)

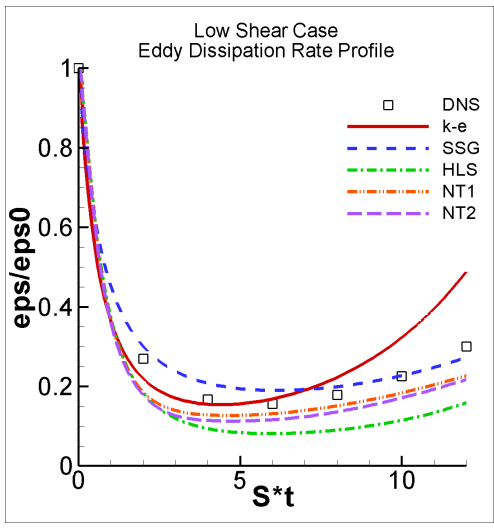

(b)

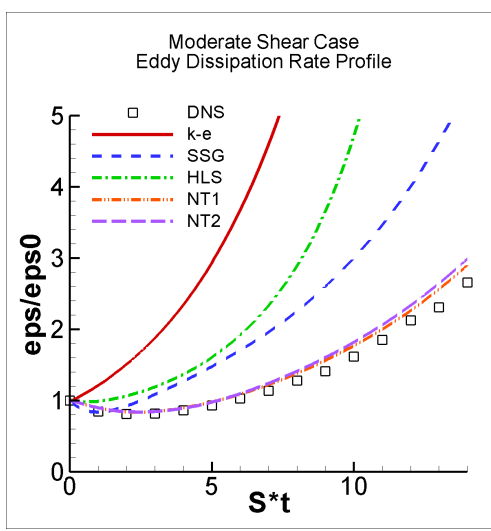

(e)

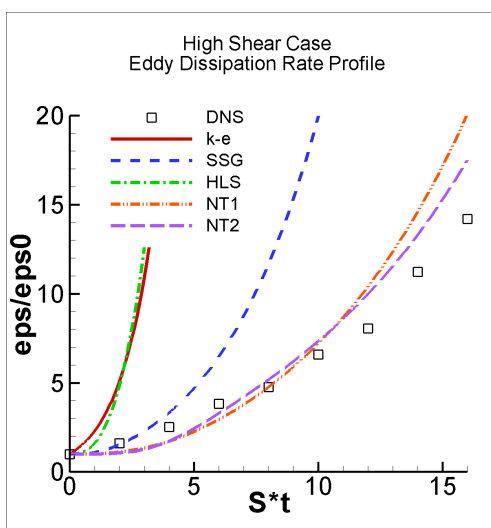

(h)

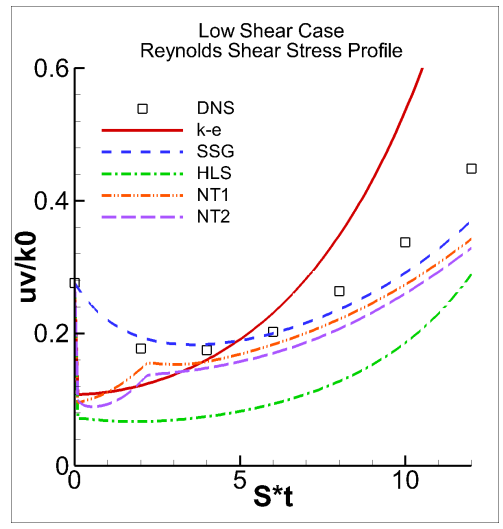

(c)

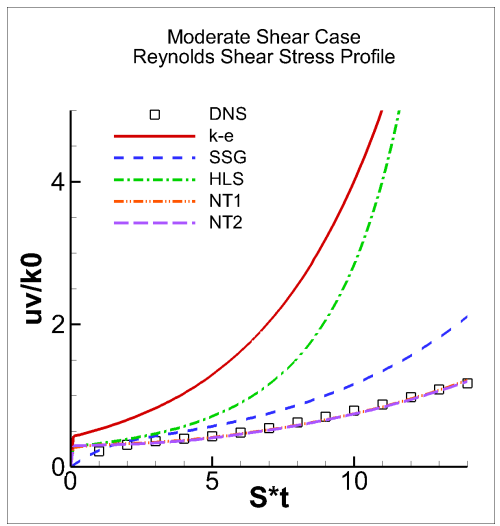

(f)

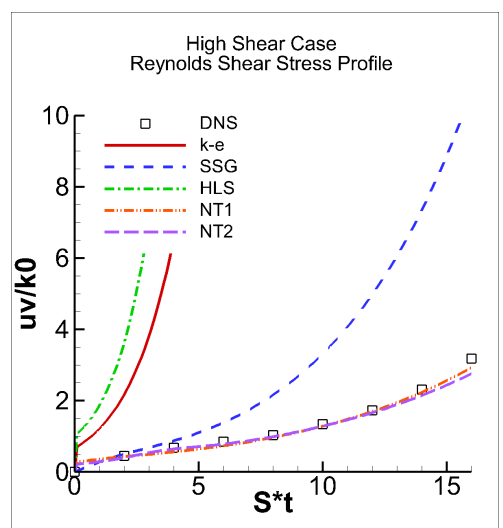

(i)

Figure 2: Prediction of the temporal evolution of the turbulent kinetic energy $k$, the eddy dissipation $\varepsilon$ and Reynolds shear stress $\overline{u v}$, all normalized by their initial values, for the homogeneous shear flow cases. (a), (b) and (c) low shear case (Rogers and Moin (1987) with $\eta_{0}=1.2$ ); (d), (e) and (f) moderate shear case (Matsumoto et al. (1991) with $\left.\eta_{0}=4.7\right)$ and; $(\mathrm{g}),(\mathrm{h})$ and (i) high shear case (Lee et al. (1990) with $\eta_{0}=16.8$ ). 


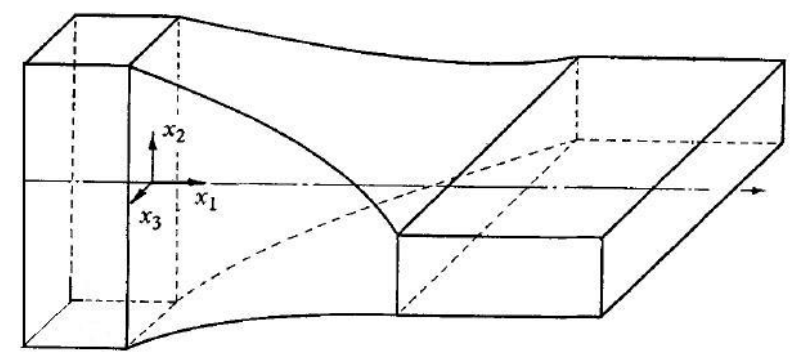

Figure 3: Normally strained flow diagram, based on Tucker and Reynolds (1968).

(1968) presents a steeper and longer change in $\eta$ than the pure plane strain case of Gence and Mathieu (1979).

The simulations focused on the central region of the duct, where immediate wall influences and viscosity effects can be neglected, the turbulence may be considered as homogeneous and the problem as unidimensional. Given that the velocity field is known and fixed, only the turbulence field equations must be solved. A simple grid with 130 control volumes in the $x$-direction has thus been used for the Tucker and Reynolds case, and a similar arrangement with 80 control volumes for the Gence and Mathieu case. The inlet conditions for the turbulent quantities have been taken from the corresponding experimental papers and the inlet value for the eddy dissipation was estimated through $k^{3 / 2} / \ell_{0}$, with the inlet length scale $\ell_{0}$ varying from 0.015 to $0.018 \mathrm{~m}$ for the Tucker and Reynolds case and from 0.01 to $0.02 \mathrm{~m}$ for the Gence and Mathieu case, depending on the turbulence model used, so that the same initial decay rate of the turbulent kinetic energy reported in the experiments was obtained.

In Figure 4, the two sets of experimental data show that the effects of normal strain on the generation rate of turbulence are not as strong as those of shear. For the type of normal straining that occurs here we have: $S=\frac{d W}{d z}=-\frac{d V}{d y}$. This results in the following expressions of the generation rates for the two normal stresses in the $x$ and $y$ direction and the turbulent kinetic energy:

$$
P_{\overline{w^{2}}}=-2 \overline{w^{2}} \frac{d W}{d z}=-2 S \overline{w^{2}} \quad P_{\overline{v^{2}}}=-2 \overline{v^{2}} \frac{d V}{d y}=2 S \overline{v^{2}} \quad P_{k}=S\left(\overline{v^{2}}-\overline{w^{2}}\right)
$$

Since the turbulence is initially isotropic, the generation rate of $k$ is initially zero, resulting in the observed initial decay of turbulence. As also indicated by the above expressions, the imposed normal strain gradually increases the normal stress in the $y$ direction and reduces that in the $z$ direction. Consequently $P_{k}$ starts to grow, until it eventually balances the dissipation rate and reverses the decay of turbulence. For the Tucker and Reynolds case in Figure 4(a), where the normal strain is weaker, the decay region extends over most of the length of the domain, while for the Gence and Mathieu case, Figure 4(b), the decay of turbulence is reversed much earlier. For the Tucker and Reynolds case, the SSG, NT1 and NT2 return the correct evolution of $k$, while the Hanjalic et al. multi-scale model returns a stronger decay of turbulence.

Since in LEV models the generation rate here will be reduced to $P_{k_{L E V}}=4 \mu_{t} S^{2}$, effective viscosity models would normally be expected to over-estimate the levels of turbulent kinetic energy. The reason why the HLS model underestimates the turbulence levels, is probably the presence of the additional source term in equation (4), which increases the dissipation rate when normal strain becomes dominant. The reason why the two multi-scale models proposed here do not over-estimate $k$ is that the normal strain causes a reduction in the value of $c_{\mu}$. The comparisons of Figure $4(\mathrm{~b})$ are entirely consistent with those of 4(a), since for this case the normal strain is stronger. 


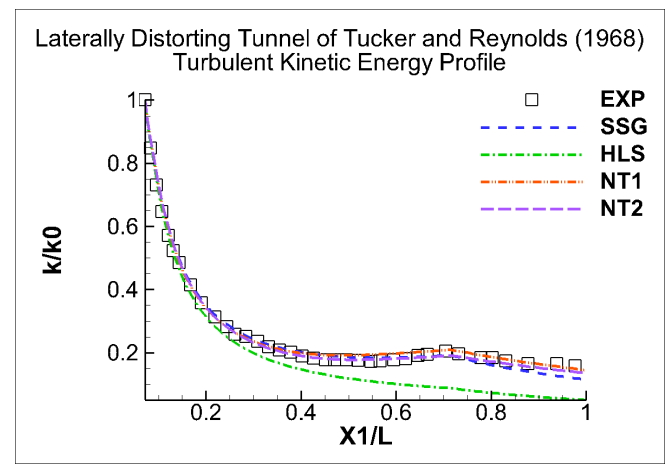

(a)

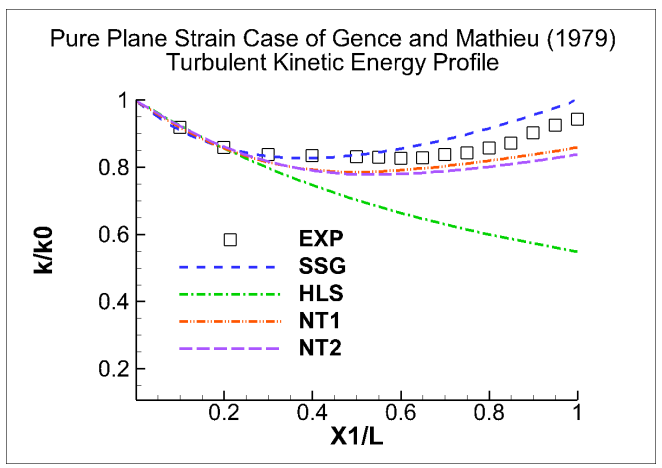

(b)

Figure 4: Profile of the turbulent kinetic energy, normalized by its inlet value $k_{0}$, in the normally strained cases with constant pure plane straining of (a)Tucker and Reynolds (1968) and (b)Gence and Mathieu (1979).

\subsection{Adverse Pressure Gradient Boundary Layer}

The performances of the NT1 and NT2 models have also been assessed in decelerating flows such as adverse-pressure-gradient boundary layers.

The adverse-pressure-gradient boundary layer of Marusic and Perry (1995) with inlet freestream velocity of $U_{\infty}=30 \mathrm{~m} / \mathrm{s}$ has been selected to be presented here. An increasing pressure has been imposed through the dimensionless parameter $C_{p}(x)=\frac{P(x)-P_{\infty}}{0.5 \rho U_{\infty}^{2}}$, where $P_{\infty}$ and $U_{\infty}$ are the inlet freestream pressure and velocity, whose variation is described by a polynomial, as a function of the streamwise coordinate $x$, as shown in Figure 5. A 3\% turbulence intensity has been used to set the inlet conditions for the turbulent quantities and a turbulent length scale of $\ell=10 \mathrm{~mm}$ has been used to calculate $\varepsilon$. The kinematic viscosity has been set to $\nu=1.58 \times 10^{-5} \mathrm{~m}^{2} / \mathrm{s}$ and a simple $5 m \times 0.2 m$ rectangular domain has been used with a $400 \times 40$ grid. The flow was allowed to develop as a zero-pressure-gradient boundary layer for $1.5 \mathrm{~m}$ so that the boundary layer thickness reached a $R e_{\theta}$ of 6430 , as reported by Marusic and Perry (1995). This case provided a Reynolds number of $R e_{x}=6.6 \times 10^{6}$, based on the free stream velocity, the kinematic viscosity and the overall length of the decelerating flow domain.

In Figure 6 the development of the turbulent kinetic energy and of the Reynolds shear stress are shown at certain downstream positions (stations T2, T5 and T6, respectively, $0.3 \mathrm{~m}$ or $R e_{x}=$ $5.7 \times 10^{5}, 1.38 \mathrm{~m}$ or $R e_{x}=2.6 \times 10^{6}$ and $1.58 \mathrm{~m}$ or $R e_{x}=3.0 \times 10^{6}$ from the point the deceleration started, as shown in Figure 5) while the pressure is increased.

The data show that the adverse pressure gradient leads to an increase in $k$ levels in the flow direction and also causes the location of maximum $k$ to be displaced further away from the wall. A similar comment can be made about the effects of adverse pressure gradients on the turbulent shear stress profiles. The cause is the thickening of the boundary layer, as the adverse pressure gradient slows down the near-wall fluid, which displaces the strong shear region away from the wall, to where the turbulence is stronger. Consequently the maximum generation rate of turbulence increases and is displaced further way from the wall. All models reproduce the overall behaviour, but with some under-prediction of the turbulent kinetic energy. While the NT1 and NT2 do not offer any predictive advantages for this case in relation to the other models, they certainly return acceptable predictions, specially considering that, since these are high-Re models, they cannot be expected to fully account for the effects of adverse pressure gradient because at least some of its influence will be on the region approximated through the use of the wall function.

\subsection{Favourable Pressure Gradient Boundary Layers}

Accelerating flows such as favourable-pressure-gradient boundary layers which reach a self-similar state have also been used as test cases. 


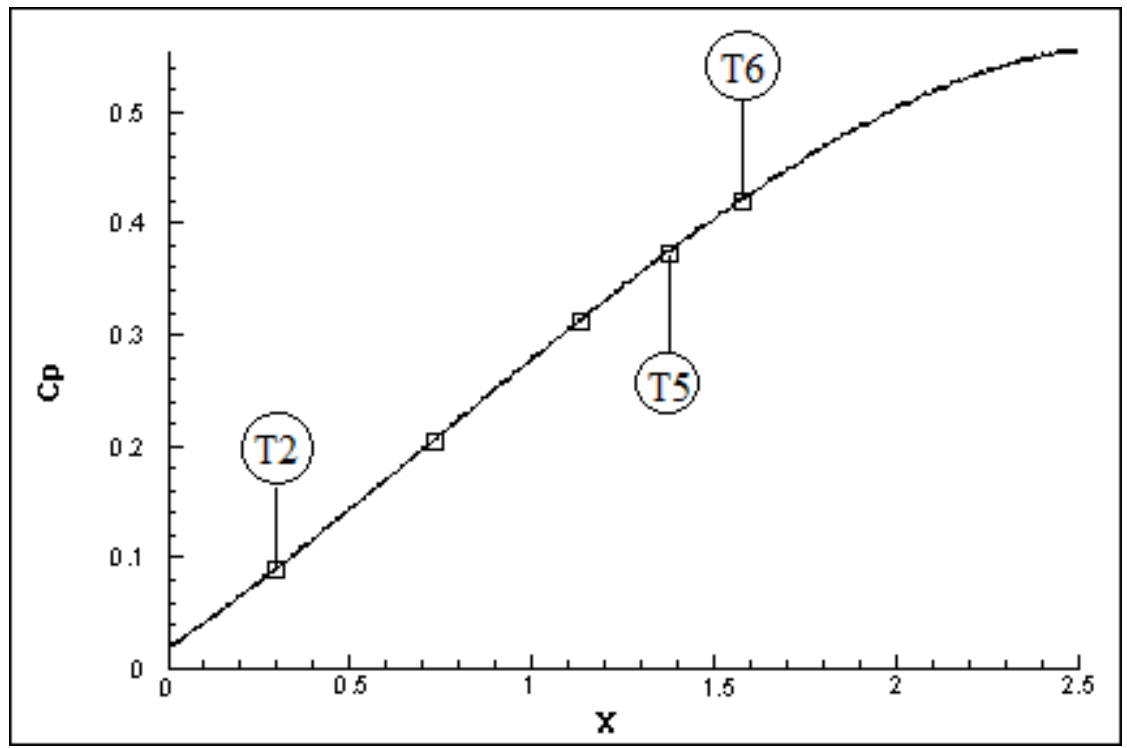

Figure 5: Dimensionless parameter $C_{P}$ as a function of the streamwise direction, with measurement stations $\mathrm{T} 2, \mathrm{~T} 4$ and $\mathrm{T} 5$ indicated for the adverse pressure gradient boundary layer case

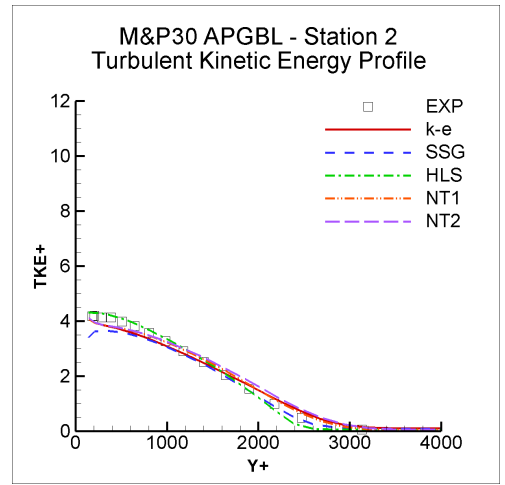

(a) station $\mathrm{T} 2$

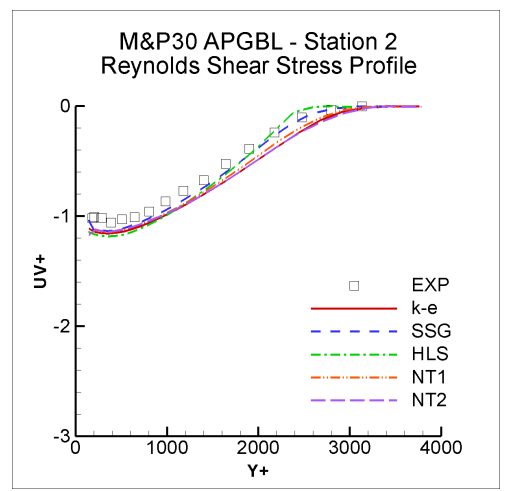

(d) station $\mathrm{T} 2$

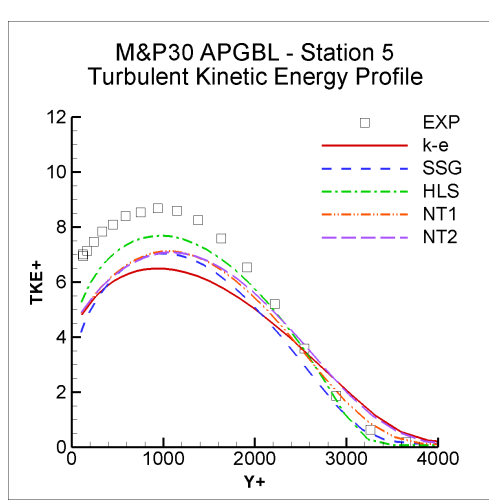

(b) station T5

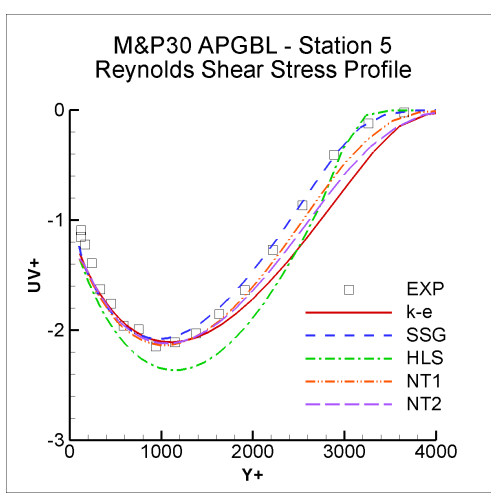

(e) station T5

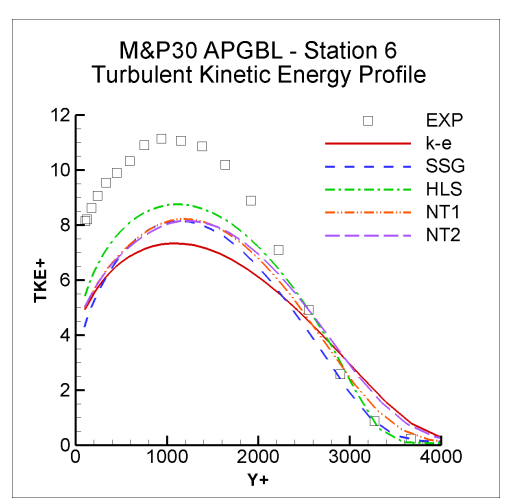

(c) station T6

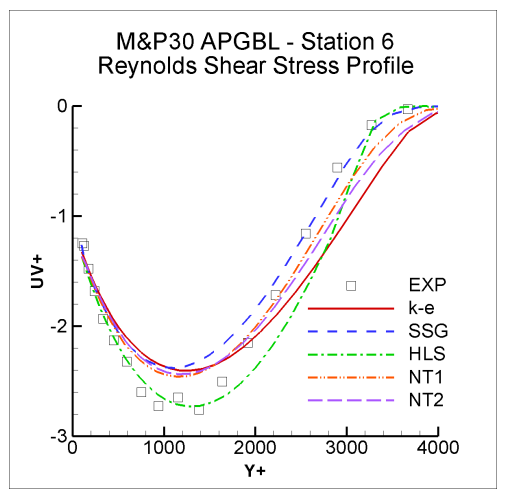

(f) station T6

Figure 6: Turbulent kinetic energy (a,b,c) and Reynolds shear stress (d,e,f) profiles at stations T2, T5 and T6 for the adverse pressure gradient boundary layer of Marusic and Perry (1995) with the highest Reynolds number. 
The sink flow cases of Spalart (1986) for only the lowest and highest values of the acceleration parameter $\left(K=\frac{\nu}{U_{\infty}^{2}} \frac{d U_{\infty}}{d x}\right)$ are presented here. Uniform profiles have been set as inlet conditions. Since comparisons with the DNS data of Spalart are for dimensionless quantities only, the exact flow properties are not relevant and reference variables such as length scale, inlet free stream velocity and density are set to unity so that the bulk Reynolds number $R e_{b u l k}$ defines the viscosity through $\mu=\frac{1}{R_{\text {bulk }}}$. Values of bulk Reynolds numbers of 87489 and 63291 have been set for the lowest and highest acceleration parameter cases respectively. An inlet turbulence intensity of $1.5 \%$ has been set for the turbulent quantities and the domain has been set as a simple rectangle, long enough to ensure the self-similar state is reached. At the upper boundary, the streamwise velocity has been determined by integrating the acceleration parameter expression, providing:

$$
U(x)=\frac{1}{\frac{1}{U_{0}}-\frac{K}{\nu} x}
$$

The imposed pressure-gradient has thus been set according to Bernoulli's equation:

$$
P_{\infty}(x)=P_{0}+\frac{\rho}{2}\left[U_{0}^{2}-U_{\infty}^{2}(x)\right]
$$

where $P_{0}$ and $U_{0}$ are the inlet freestream pressure and velocity respectively.

The profiles of mean velocity, turbulent kinetic energy and Reynolds shear stress for the lowest and highest acceleration parameter cases are presented in Figure 7. It can be noted that the velocity profile lies above the wake part of the log law and tends to deviate further away as the acceleration parameter increases. The turbulent quantities' peaks decrease near the wall and their magnitude decays faster toward the outer layer as the acceleration increases, which is consistent with the tendency of laminarization of favourable-pressure-gradient boundary layer flows.

The lower the acceleration parameter, the closer to a zero-pressure-gradient boundary layer the flow is and therefore the better is the performance of the turbulence models as a whole, which, as discussed in Klein et al. (2015), follow the correct trend, but not enough in quantitative terms. The same can be said of both NT1 and NT2 models which perform similarly to the high-Re $k-\varepsilon$ and SSG models, returning reasonable and acceptable results, which confirms their appropriate tuning. As was also noted in the discussion of the boundary layer flows subjected to adverse pressure gradients in section 3.3, all models tested here use the wall-function approach and consequently do not resolve the viscous sub-layer. Consequently their ability to reproduce the effect of the pressure gradient on the thickness of the viscous sub-layer is rather limited. The reason why the HLS model seems to perform best is probably due to the fact that in low homogeneous shear this model under-estimates turbulence levels. This predictive flaw therefore cancels out the error caused by the wall-function approximations.

\subsection{Oscillatory Boundary Layer}

Oscillatory boundary layers, achieved by imposing an oscillatory freestream pressure gradient, provide characteristics of both adverse- and favourable-pressure-gradient boundary layers and yet vary in time.

The experimental case of Jensen et al. (1989) performed in a smooth bed with $R e_{a}=6 \times 10^{6}$ ( $R e_{a}$ defined as $R e=\frac{a U_{0 \infty}}{\nu}, a=\frac{U_{0 \infty}}{\omega}$ ) has been selected to assess the performance of the turbulence models under investigation. In order to apply an oscillatory freestream pressure, the $x$-directed pressure gradient has been specified to oscillate periodically as $\frac{d P}{d x}=d P_{a m p} \sin (\omega t)$, where $\omega=2 \pi / T$ and $d P_{a m p}=4 / 3 \mathrm{~Pa} / \mathrm{m}$ is the amplitude magnitude of the pressure gradient which ensures the free-stream velocity oscillates sinusoidally with a period of $T=9.72 \mathrm{~s}$ about a zero-mean value and amplitude of $U_{0 \infty}=2 \mathrm{~m} / \mathrm{s}$. A simple 1D domain in the $y$ direction could be employed with Neumann boundary conditions for all variables and only the streamwise direction momentum equation and the turbulence closure equations needed to be solved. A total of 56 control volumes in the $y$ direction discretised the domain and the time step has been set by dividing the period of oscillation by 240 . 


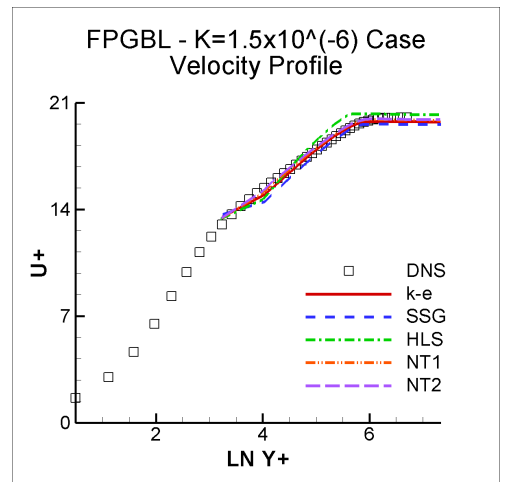

(a) $K=1.5 \times 10^{-6}$

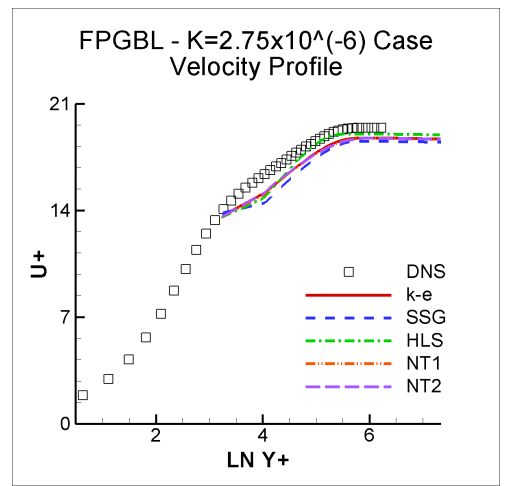

(d) $K=2.75 \times 10^{-6}$

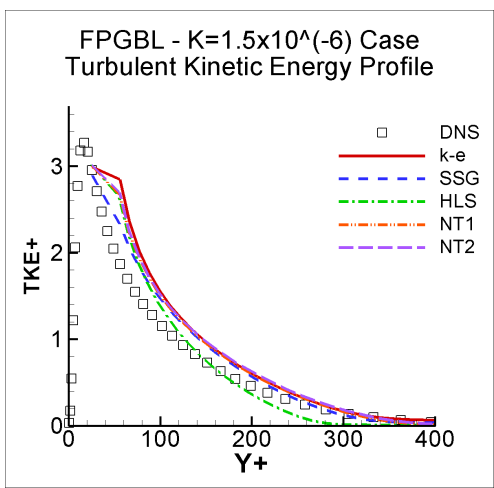

(b) $K=1.5 \times 10^{-6}$

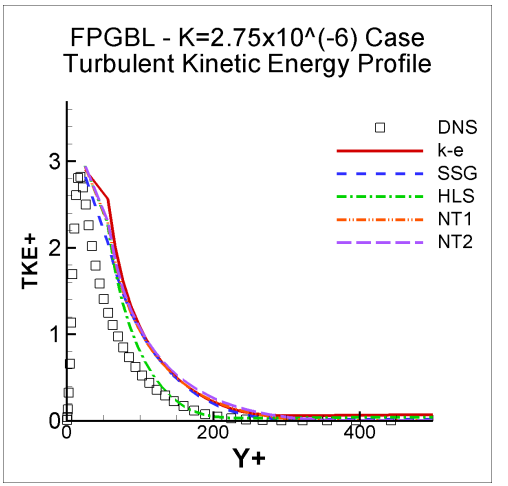

(e) $K=2.75 \times 10^{-6}$

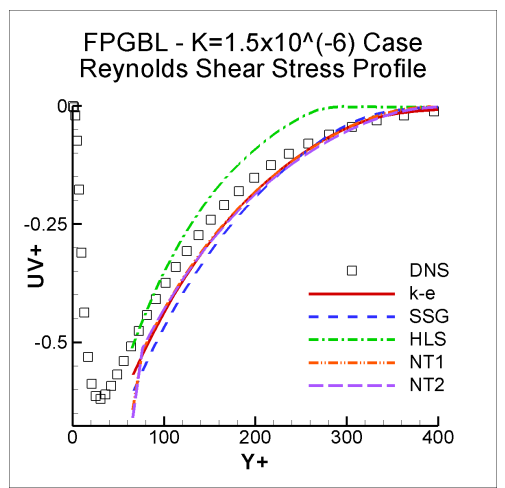

(c) $K=1.5 \times 10^{-6}$

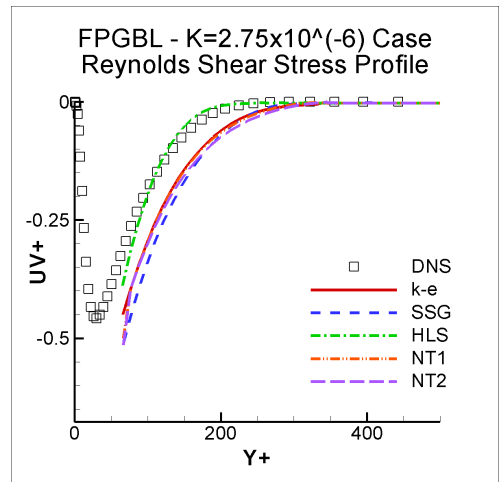

(f) $K=2.75 \times 10^{-6}$

Figure 7: Mean velocity, turbulent kinetic energy and Reynolds shear stress profiles in the favourable pressure gradient boundary layer of Spalart (1986) with (a), (b) and (c) $K=1.5 \times 10^{-6}$ and (d), (e) and (f) $K=2.75 \times 10^{-6}$. 


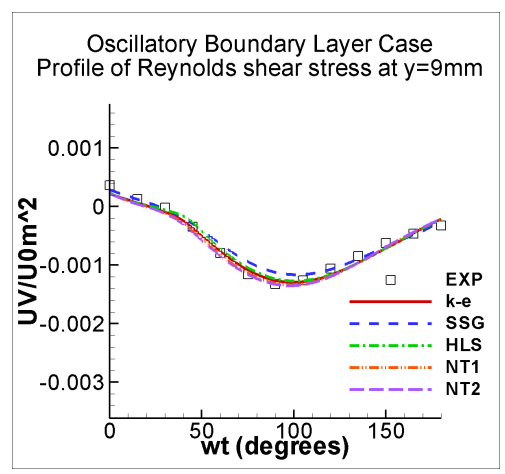

(a) $y=9 m m\left(y^{+} \approx 750\right)$

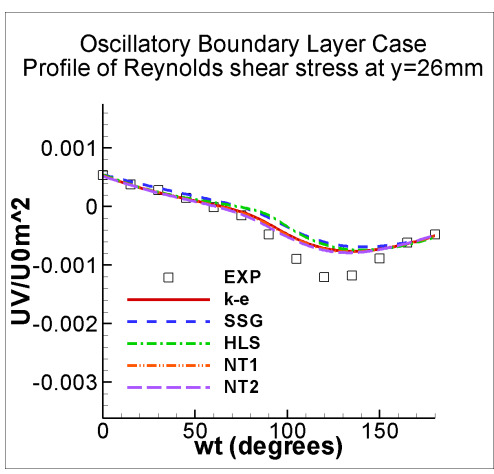

(b) $y=26 m m\left(y^{+} \approx 2200\right)$

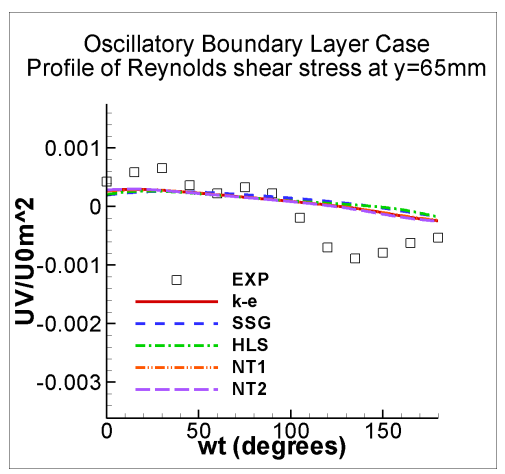

(c) $y=65 \mathrm{~mm}\left(y^{+} \approx 5300\right)$

Figure 8: Prediction of the temporal variation of the Reynolds shear stress in the oscillatory boundary layer case of Jensen et al. (1989) at three distances from the wall.

The time-history of the Reynolds shear stress is shown for three locations across the boundary layer in Figure 8. It can be noted that whereas the experiments show a cyclic behaviour up to the outer region of the oscillating boundary layer, the turbulence models erroneously predict very little temporal variation for the Reynolds shear stress far from the near-wall region, characteristic of a frozen turbulence field. The NT1 and NT2 models are no exception to this, however, like the other models, they return reasonable predictions, especially at locations closer to the wall. As discussed in Klein et al. (2015), the turbulence models tend to predict the response of the turbulence field to the mean flow oscillation to decrease with wall distance rather faster than in the experiments.

\subsection{Oscillatory Pipe Flows}

An oscillatory pipe flow case, where a periodically oscillating bulk velocity is imposed with a frequency in the intermediate range, where non-equilibrium features are expected, has been chosen to evaluate the performance of the NT1 and NT2 models here. The experimental test case of He and Jackson (2009) with a bulk Reynolds number of $R e=14000$, amplitude of oscillation of $A=20 \%$ and period of $T=3.14 \mathrm{~s}$ has been selected to be discussed here. This case is characterized by $\omega^{+}=\frac{\omega \nu}{\bar{u}_{\tau}^{2}} \approx 0.007$, which means it is within an intermediate frequency range where non-equilibrium features in the response of the turbulence are expected (Klein et al., 2015).

Profiles from a fully developed steady state pipe flow have been applied as initial conditions. The domain, axisymmetric and in the cylindrical-polar coordinate system, extended from $y=0$ to $y=R, R=0.0254 m$ being the pipe's radius. The length of the domain is not relevant, since periodic boundary conditions in the flow direction have been applied to ensure a fully developed pipe flow. The grid consists of 25 control volumes in the $y$ direction and the time step has been set to provide 160 steps within one full cycle of oscillation.

In order to compare experimental and numerical results, the first harmonic of the turbulent quantities $\left(<q(t, y)>=<q(y)>\left[1+A_{q} \cos \left(\omega t-\phi_{q}\right)\right]\right)$ has been employed, $<q(y)>A_{q}$ and $\phi_{q}$ denoting the amplitude and phase shift of the turbulent quantity $q$ respectively.

In Figure 9 the amplitude and phase shift of mean velocity and Reynolds shear stress are shown. As can be seen in Figure 9, for the velocity, the amplitude is largely uniform over the pipe radius, with a modest maximum near the wall. The velocity phase shift is uniform at the pipe core at around $5^{\circ}$ lag due to the high inertia, and then falls over the outer half of the pipe to $-10^{\circ}$ lead near the wall, where the fluid has little inertia. The amplitude of the turbulent shear stress fluctuations on the other hand, increases, almost linearly, from zero at the centre, where its mean value is also zero, to a maximum near the wall. The shear stress phase shift, on the other hand, shows a strong lag close to the pipe centre, where the actual value is zero, which falls to zero at the wall, making the wall shear stress in phase with the bulk velocity. All models return very similar characteristics for the velocity field, which are close to the measured data. For the shear stress characteristics, there 


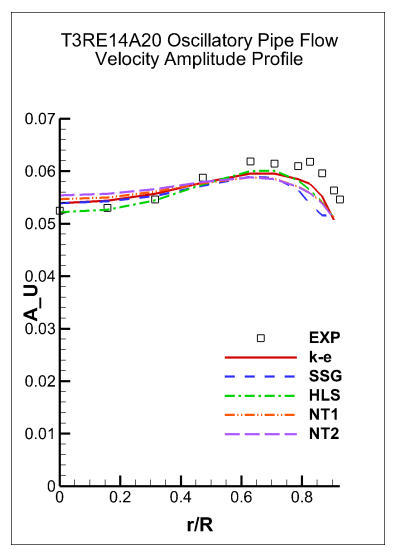

(a) $U$ amplitude

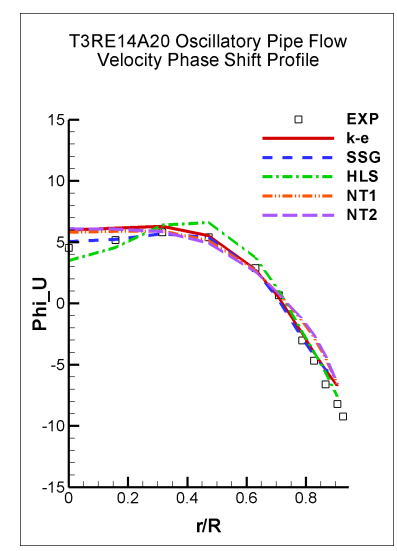

(b) $U$ phase shift

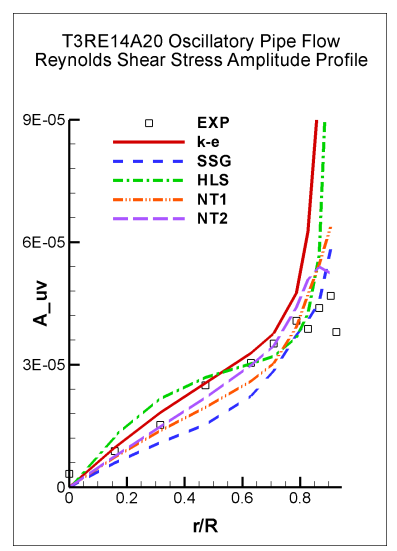

(c) $\overline{u v}$ amplitude

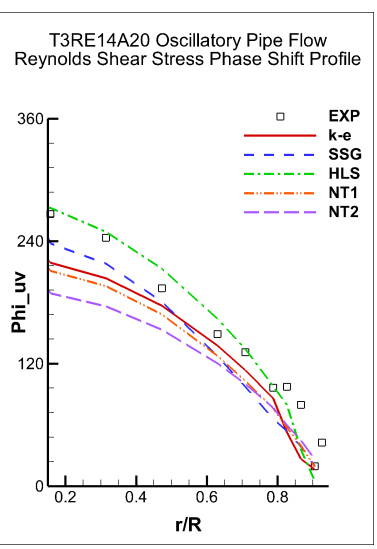

(d) $\overline{u v}$ phase shift

Figure 9: Prediction of the radial variation of the velocity and Reynolds shear stress amplitude and phase shift for the oscillatory pipe flow case of He and Jackson (2009) with $R e=14000, A=20 \%$ and $T=3.14 s$.

is a noticeable deviation in the predictions of the different models. For the amplitude variation the NT1 and NT2 predictions are in close accord with the data. The large phase lag in the shear stress oscillations at the pipe centre is under-estimated, but given that the amplitude goes to zero at this region, the uncertainty in the measured values of the phase shift must be high.

One feature to emerge from comparisons from this case (Figure 9), the adverse-pressure-gradient (Figure 6) and the favourable-pressure-gradient (Figure 7) boundary layer cases, is that the HLS model seems to perform slightly better than the other models assessed here. However, the coefficients of the HLS model have been found not to satisfy completely the constraint equations of asymptotic analyses carried out (Klein, 2012). This suggests that this better prediction in these cases may be caused by a shift in the coefficients from their equilibrium values.

\subsection{Ramp Up Fully Developed Pipe Flow}

This case involves a linear increase in time in the bulk velocity of a fully developed pipe flow. In order to assess the performance of the turbulence models, the experimental work of He and Jackson (2000) has been used. The case for which they provided time histories of all turbulence quantities, except the eddy-dissipation rate, at four radial locations has been selected. In this case, the bulk Reynolds number of the flow is linearly increased from 7000 to 45200 over a period of 5 seconds.

This case has been set up similarly to the oscillatory pipe flow case: axisymmetric, in a cylindrical-polar coordinate system with $y$ varying from $y=0$ to $y=R=0.0254 m$, initial conditions from a fully developed pipe flow, periodic boundary conditions in the flow direction to ensure the fully developed state of the flow and a grid with 25 control volumes in the $y$ direction.

It can be noted in Figure 10 that the turbulent kinetic energy and the Reynolds shear stress show a lag in time before responding to the imposed change in the bulk velocity, especially near the centre, where as also noted in the discussion of the previous case, the inertia of the fluid is greater, thus showing clear non-equilibrium features of this flow.

As noted by Klein et al. (2015), the turbulence models tend to either predict the correct profile of the time history of the turbulent quantities at the centre of the pipe or at a mid-distance from the pipe wall and its centre. The same happens with the NT1 and NT2 models, which best reproduce the response of the turbulence field to the changes in the mean flow closer to the pipe walls, with greater, though still relatively minor, deviations from the measured behaviour at the pipe centre. They do not perform poorly in the centre of the pipe, and indeed predict correctly the moment the turbulence starts to respond, however they tend to slightly overpredict the turbulent kinetic energy in this region as this quantity builds up. The standard $k-\varepsilon$ and SSG models tend to do the opposite, predicting the flow best in the centre of the pipe. Either way, these four models all 


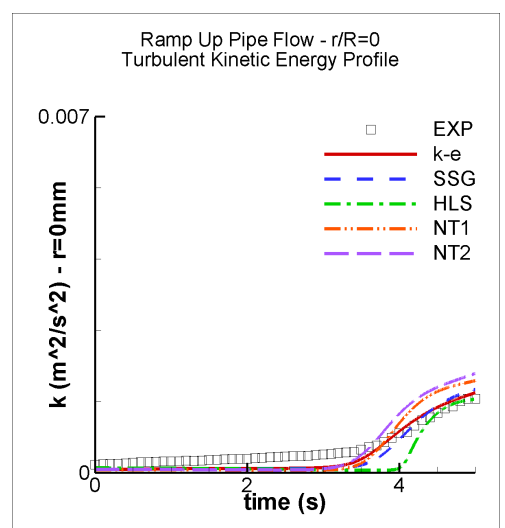

(a) $r / R=0$

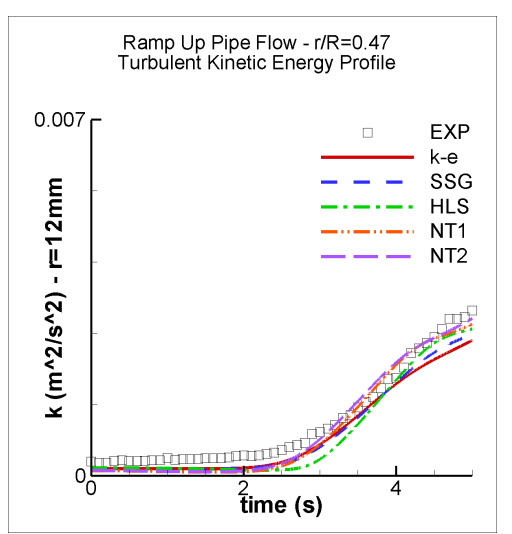

(b) $r / R=0.47$

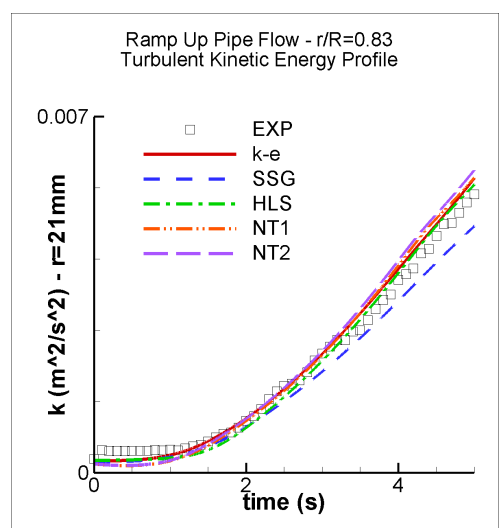

(c) $r / R=0.83$

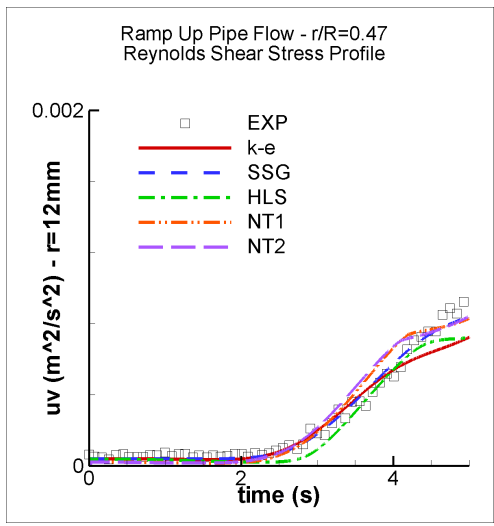

(d) $r / R=0.47$

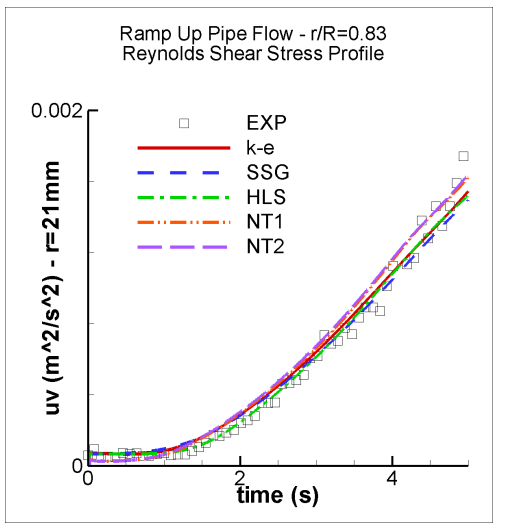

(e) $r / R=0.83$

Figure 10: Temporal evolution of the turbulent kinetic energy and Reynolds shear stress profiles at selected $y$ positions in the fully developed ramp up pipe flow case of He and Jackson (2000).

return reasonable and acceptable results. In this case though, the HLS model predicts too large a delay in the moment the turbulent kinetic energy starts to respond to the changes in the mean flow.

\subsection{Backward Facing Step Flows}

These are essentially flows in channels which undergo a sudden expansion as shown in Figure 11. As also included in Figure 11, the experimental flows simulated here feature an oscillating jet at the corner of the step in order to generate a pulsated flow over the backward facing step. A strongly non-equilibrium shear region is formed where curved streamlines and recirculation are confined between the step wall and the reattachment point. An important parameter to be predicted in such cases is the reattachment length and its response to pulsation.

The experimental work of Chun and Sung (1996) has been used to assess the turbulence models. The studied cases are at a Reynolds number $R e_{H}$, based on the step height $H$ and bulk velocity $U_{0}$ just before the step $(x / H=-0.02)$ equal to 33000 . Both steady and unsteady state simulations have been performed, the latter driven by an oscillatory jet placed at the step corner with forcing frequencies characterized by the Strouhal number, $S t=\frac{f H}{U_{0}}$. In all cases the domain was the same, being a 2-D channel with expansion ratio of 1.5. A developing channel flow profile has been used as inlet for the steady state case in order to match experimental inlet conditions and the steady state case has subsequently been used as initial/inlet condition for the unsteady ones. A bulk pressure correction has been applied at the outlet to enhance numerical stability by imposing global continuity. The grid employed in the computations consisted of 180x70 nodes in the axial and cross-channel directions respectively and the time step was set by dividing the cycle into 400 


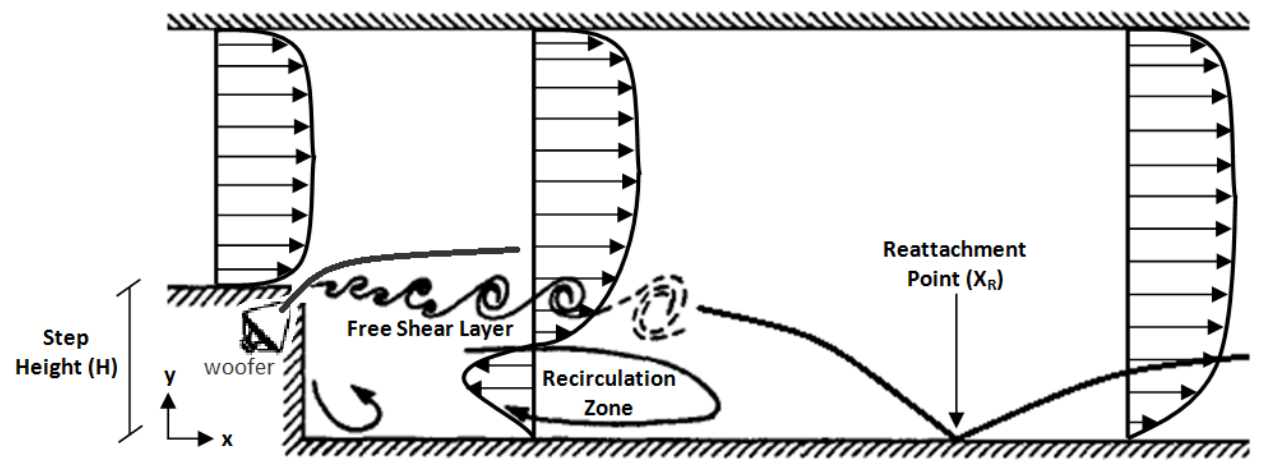

Figure 11: Backward facing step sketch, adapted from Simpson (1996) and Chun and Sung (1996).

steps.

As can be noted in Figure 12, initially the reattachment length tends to decrease as the frequency of oscillation increases, reaching a minimum value of about $65 \%$ that of the stationary case at $S t=0.275$ and then starting to increase again until it reaches a value similar to that for the steady state case, when $S t$ becomes greater than 1. As reported by Klein et al. (2015), although the turbulence models generally tend to follow this trend, they tend either to predict correctly the reattachment length in steady state cases, but not to return the strong reduction of reattachment length with oscillation frequency, or to under-predict the reattachment length in steady state cases and end up predicting correctly the reattachment length at the frequency where the time-averaged reattachment length reaches a minimum.

Figure 12 compares the variations of time-averaged reattachment lengths predicted by the proposed NT1 and NT2 models and also those predicted by the reference models $k-\varepsilon$ and SSG, with the corresponding experimental variation, provided by Chun and Sung (1996). The two-timescale models proposed here are clearly the only models able to follow the correct trend and reach the extreme values, that is at the steady state and high frequency cases (where the time-averaged reattachment length is maximum) and at the frequency where the time-averaged reattachment length is minimum.

The HLS model presented stability problems in this case, and only converged for the steady state flow. These problems have been traced to the extra source term identified and discussed earlier in its its $\varepsilon_{P}$ equation. Although the NT2 model retains this term, its coefficient is lower than that used in the HLS model and no stability problems have been encountered with it.

In Table 4 the time-averaged reattachment lengths predicted by the five turbulence models considered here are reported for three oscillation frequencies, expressed in a non-dimensional form through the Strouhal number St. These were actually the three cases used for the tuning of the extra terms $f_{B L}$ in the NT1 and NT2 models. It should be noted that although only three cases were considered for the tuning process, the physical effects seem to have been captured by the expressions proposed, since the prediction for all the other oscillation frequencies, shown in Figure 12 , are reasonable and the reattachment length is generally captured as a function of the oscillation frequency.

Table 4 shows that both the standard $k-\varepsilon$ and SSG models underpredict the steady state reattachment length. While the $k-\varepsilon$ model manages to reduce the reattachment length to the correct value at $S t=0.275$, at a higher frequency case $(S t=1)$ it can not increase it enough. The SSG model does not reduce the reattachment length enough at $S t=0.275$, but increases this parameter relatively well at $S t=1$. The HLS model clearly under-predicts the reattachment length at the steady state. Both proposed models, NT1 and NT2, provide good results at all frequencies.

The mean velocity profiles are shown in Figure 13 for the three cases reported in Table 4. It can be seen that, overall, the NT1 and NT2 models return the profiles closest to those of the experiments. Although they predict the correct reattachment length in these three cases, the profiles immediately after the reattachment point are not in complete accord with the measurements. 


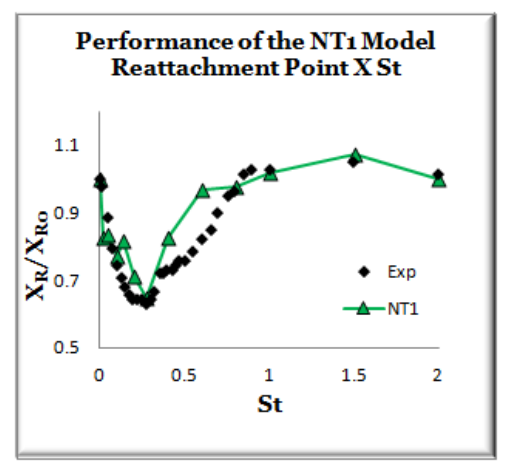

(a)

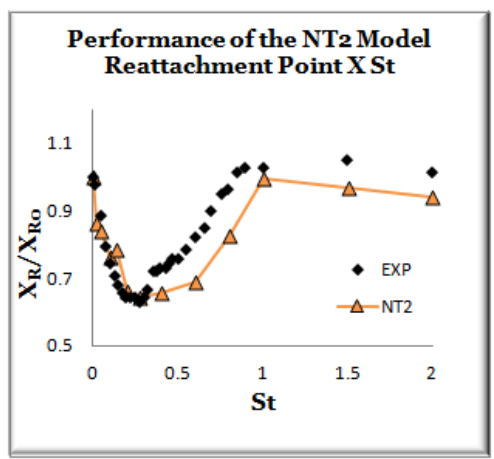

(b)

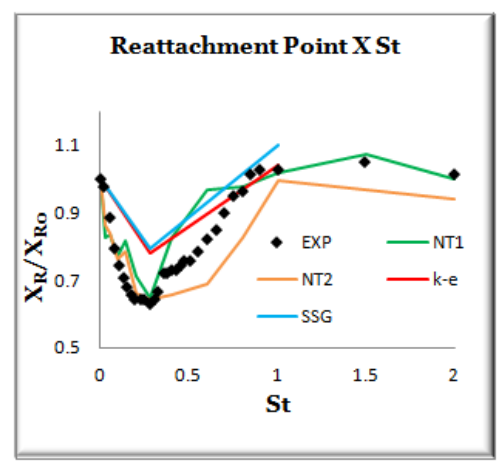

(c)

Figure 12: Prediction of the time-averaged reattachment point, normalized by the reattachment point predicted for the steady state case, in unsteady backward facing step cases with different oscillation frequencies. Experimental data from Chun and Sung (1996). (a)Performance of the NT1 model. (b) Performance of the NT2 model. (c) Comparison between the performance of the NT1 and NT2 models with the standard $k-\varepsilon$ and SSG models.

The reason for this divergence between the predicted and measured profiles, which does diminish further downstream, as discussed in Klein et al. (2015), is the slow boundary layer recovery after reattachment presented by turbulence models. In order to address this issue, the prediction of turbulence models in developing boundary layers needs to be further explored.

Table 4: Comparison of the prediction of the normalized time-averaged reattachment length $\left(X_{R} / H\right)$ in the three backward facing step cases.

\begin{tabular}{cccc}
\hline \hline Model/Experiment & $\mathrm{St}=0$ & $\mathrm{St}=0.275$ & $\mathrm{St}=1$ \\
\hline Experiment & 7.8 & 5.0 & 8.1 \\
$k-\varepsilon$ & 6.42 & 5.01 & 6.67 \\
$\mathrm{SSG}$ & 6.95 & 5.51 & 7.66 \\
$\mathrm{HLS}$ & 4.14 & - & - \\
$\mathrm{NT} 1$ & 7.82 & 5.05 & 7.96 \\
$\mathrm{NT} 2$ & 7.84 & 5.05 & 7.81 \\
\hline
\end{tabular}

\section{Concluding Remarks}

Two new two-time-scale linear-eddy-viscosity models, the NT1 and NT2, have been developed using the Hanjalic et al. (1980) version as the starting point, with non-equilibrium turbulent flows very much in mind. They have been shown to combine physical fidelity with numerical robustness.

The primary difference between these two new models is in the eddy viscosity formulation. Asymptotic analyses of homogeneous shear flows, decaying grid turbulence and local equilibrium boundary layer flows have been used to determine the coefficients of the terms appearing in these models and also to assess their consistency with the turbulent kinetic energy spectrum. Further differences between the development of the two-time-scale models proposed here and that of models of the same type from earlier studies, arise from making the turbulent viscosity dependent on the strain rate, a feature introduced earlier in Craft et al. (1996) and Craft et al. (1999) for single-scale models, and also from exploiting the additional information available on the turbulence spectrum, to make the model sensitive to non-equilibrium flow features.

The performances of these two-scale models (NT1 and NT2) have been compared to those of three other models; the two-time-scale linear-eddy-viscosity model of Hanjalic et al. (1980), the widely used standard $k-\varepsilon$ model and the single-time-scale Reynolds-stress-transport model of 


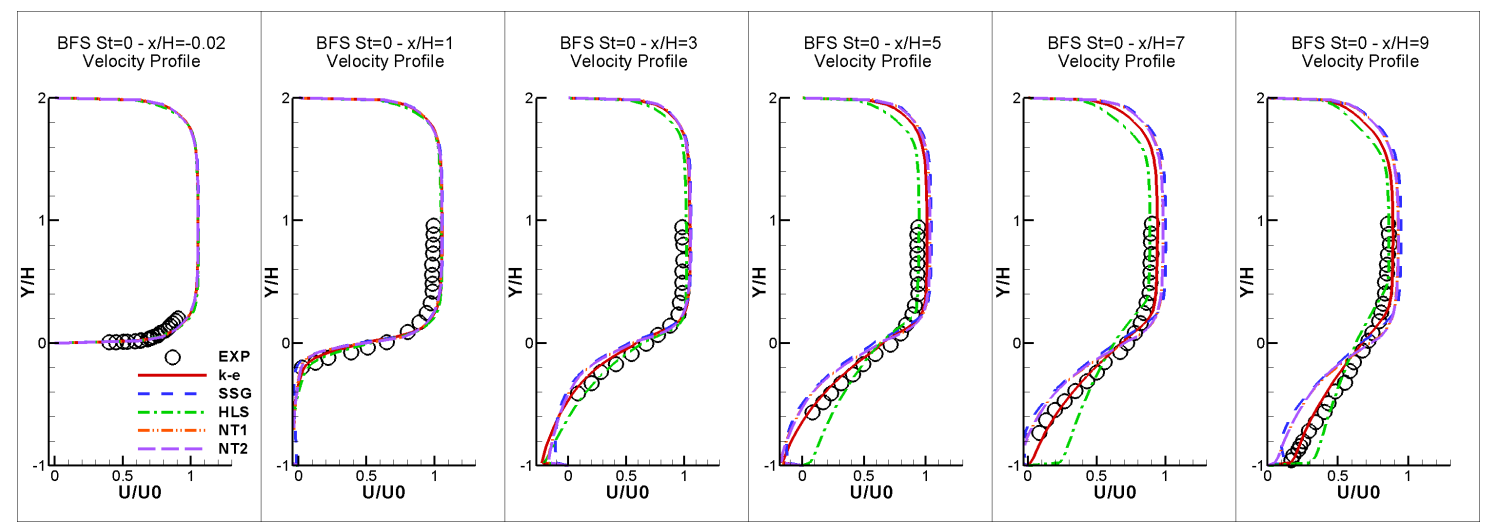

(a) Steady State

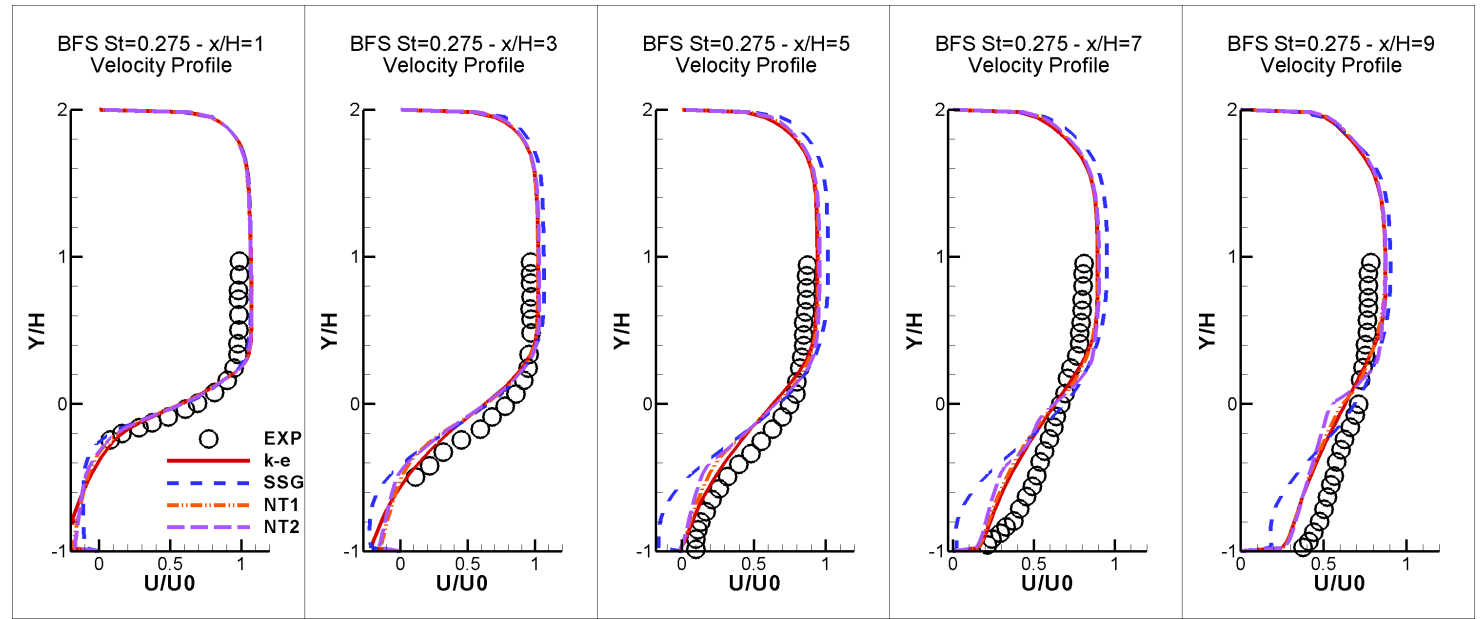

(b) $S t=0.275$

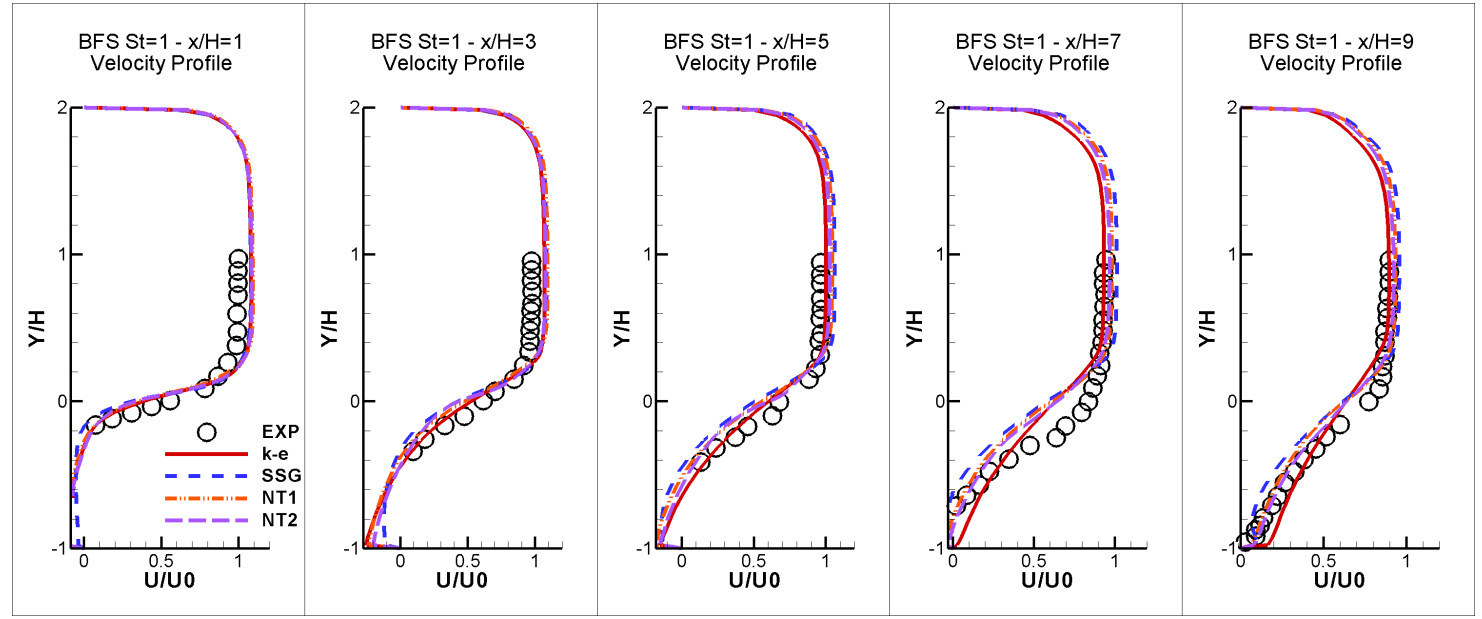

(c) $S t=1$

Figure 13: Time-averaged velocity profile in the backward facing step flow cases of Chun and Sung (1996). (a) steady state case. (b) $S t=0.275$. (c) $S t=1$. 
Speziale et al. (1991), referred to as the SSG model, which is a more complex scheme than the linear-eddy-viscosity approach of the other models mentioned. The NT1 and NT2 models provide clear improvements in predicting a wide range of homogeneous shear flows, even compared to the second-moment SSG model, which usually performs well in such flows. Improvements are also noted in the prediction of the turbulent kinetic energy in normally strained cases, where lineareddy-viscosity models are not expected to return good predictions. The proposed two-time-scale models have also proved capable of predicting the dependence of the re-circulation length on the forcing frequency in pulsated flows over a backward facing step. None of the other 14 models tested in Klein et al. (2015) are capable of such prediction. In the other test cases presented, the two two-time-scale models developed here performed reasonably well, returning predictions similar to those of the standard $k-\varepsilon$ and the SSG. The NT1 and NT2 models have thus been validated over a wide range of equilibrium and non-equilibrium flows.

It is also important to highlight that these two-time-scale models have proved to be robust to employ, with processor time requirements similar to those of the standard $k-\varepsilon$.

As far as modelling is concerned, it is noted that the models which satisfy the asymptotic states of simple flows tend not to be able to capture effects of strongly non-equilibrium flows. In this context, working with two-time-scale models proved to be a more flexible approach, since spectral ratios such as $\frac{k_{P}}{k_{T}}$ and $\frac{\varepsilon_{P}}{\varepsilon_{T}}$ are available for modelling purposes.

It can thus be concluded that the two-time-scale models developed here emerge as good alternatives to improve the prediction of non-equilibrium shear dominated flows, using a rather simple formulation still based on linear-eddy-viscosity approaches. Further development of MTS models, exploring the presence of the spectral ratios $\frac{k_{P}}{k_{T}}$ and $\frac{\varepsilon_{P}}{\varepsilon_{T}}$, can be extended to low-Reynolds-number approaches, non-linear-eddy-viscosity schemes and second-moment-closure models, where changes in the spectral shape can be taken into account for the individual stresses.

\section{Acknowledgments}

The authors acknowledge the support of the School of Mechanical Aerospace and Civil Engineering for the Overseas Research Student scholarship and CNPq, National Council for Scientific and Technological Development - Brazil, for the PhD scholarship.

\section{References}

A. Cadiou, K. Hanjalic, and K. Stawiarski. A two-scale second-moment turbulence closure based on weighted spectrum integration. Theoret. Comput. Fluid Dynamics, 18:1-26, 2004.

C. P. Chen and K. L. Guo. A non-isotropic multiple-scale turbulence model. Applied Mathematics and Mechanics, 12:981-991, 1991.

C. C. Chieng and B. E. Launder. On the calculation of turbulent heat transport downstream from an abrupt pipe expansion. Numerical Heat Transfer, Part A: Applications, 3:189-207, 1980.

K. B. Chun and H. J. Sung. Control of turbulent separated flow over a backward-facing step by local forcing. Experiments in Fluids, 21:417-426, 1996.

T. J. Craft, B. E. Launder, and K. Suga. Development and application of a cubic eddy-viscosity model of turbulence. International Journal of Heat and Fluid Flow, 17(2):108-115, 1996.

T. J. Craft, H. Iacovides, and J. H. Yoon. Progress in the use of non-linear two-equation models in the computation of convective heat-transfer in impinging and separated flows. Flow, Turbulence and Combustion, 63:59-80, 1999.

B. S. Duncan, W. W. Liou, and T. H. Shih. A multiple-scale turbulence model for incompressible flow. NASA Technical Memorandum 106113, 1993. 
J. N. Gence and J. Mathieu. On the application of successive plane strains to grid-generated turbulence. J. Fluid Mech., 93:501-513, 1979.

M. M. Gibson and B. E. Launder. Ground effects on pressure fluctuations in the atmospheric boundary layer. J. Fluid Mech., 86:491-511, 1978.

K. Hanjalic, B. E. Launder, and R. Schiestel. Multiple-time-scale concepts in turbulent transport modelling. In Turbulent Shear Flow 2, Berlin, July 2-4 1980. Springer-Verlag.

S. He and J. D. Jackson. A study of turbulence under conditions of transient flow in a pipe. $J$. Fluid Mech., 408:1-38, 2000.

S. He and J. D. Jackson. An experimental study of pulsating turbulent flow in a pipe. European Journal of Mechanics B/Fluids, 28:309320, 2009.

B. L. Jensen, B. M. Sumer, and J. Fredsoe. Turbulent oscillatory boundary layers at high Reynolds numbers. J. Fluid Mech., 206:265-297, 1989.

W . P. Jones and B. E. Launder. The prediction of laminarization with a two-equation model of turbulence. Int. J. Heat Mass Transfer., 15:301-314, 1972.

H. S. Kang, S. Chester, and C. Meneveau. Decaying turbulence in an active-grid-generated flow and comparisons with large-eddy simulation. J. Fluid Mech., 480:129-160, 2003.

S. W. Kim. Calculation of divergent channel flows with a multiple-time-scale turbulence model. AIAA Journal, 29:547-554, 1991.

S. W. Kim and T. J. Benson. Calculation of a circular jet in crossflow with a multiple-time-scale turbulence model. International Journal of Heat and Mass Transfer, 35(10):2357-2365, 1992.

S. W. Kim and C. P. Chen. A multiple-time-scale turbulence model based on variable partitioning of the turbulent kinetic energy spectrum. Numerical Heat Transfer, 16:193-211, 1989.

T. S. Klein. The Development and Application of Two-Time-Scale Turbulence Models for NonEquilibrium Flows. PhD thesis, School of MACE, The University of Manchester, 2012.

T. S. Klein, T. J. Craft, and H. Iacovides. Assessment of the performance of different classes of turbulence models in a wide range of non-equilibrium flows. International Journal of Heat and Fluid Flow, 51:229-256, 2015.

B. E. Launder and D. B. Spalding. The numerical computation of turbulent flows. Computer Methods in Applied Mechanics and Engineering, 3(2):269-289, 1974.

M. J. Lee, J. Kim, and P. Moin. Structure of turbulence at high shear rate. J. Fluid Mech, 216: 561-583, 1990.

F. S. Lien and M. A. Leschziner. A general non-orthogonal collocated finite volume algorithm for turbulent flow at all speeds incorporating second-moment turbulence-transport closure, part 1: Computational implementation. Computer Methods in Applied Mechanics and Engineering, 114: 123-148, 1994.

I. Marusic and A. E. Perry. A wall-wake model for the turbulence structure of boundary layers. part 2. further experimental support. J. Fluid Mech, 298:389-407, 1995.

A. Matsumoto, Y. Nagano, and T. Tsuji. Direct numerical simulation of homogeneous turbulent shear flow. 5th Symposium on Computational Fluid Dynamics, Tokyo, 1991.

Y. Nagano, M. Kondoh, and M. Shimada. Multiple time-scale turbulence model for wall and homogeneous shear flows based on direct numerical simulations. International Journal of Heat and Fluid Flow, 18(4):346-359, 1997. 
S. B. Pope. Turbulent Flows. Cambridge University Press, 2000.

C. M. Rhie and W. L. Chow. Numerical study of the turbulent flow past an airfoil with trailing edge separation. AIAA Journal, 21:1525-1535, 1983.

M. M. Rogers and P. Moin. The structure of the vorticity field in homogeneous turbulent flows. $J$. Fluid Mech, 176:33-66, 1987.

R. Rubinstein. Formulation of a two-scale model of turbulence. ICASE Report No. 2000-5, 2000.

R. L. Simpson. Aspects of turbulent boundary-layer separation. Prog. Aerospace Sci., 32:457-521, 1996.

P. R. Spalart. Numerical study of sink-flow boundary layers. J. Fluid Mech, 172:307-328, 1986.

C. G. Speziale. Analytical methods for the development of Reynolds-stress closures in turbulence. Annual Review of Fluid Mechanics, 23(1):107-157, 1991.

C. G. Speziale, S. Sarkar, and T. B. Gatski. Modelling the pressure-strain correlation of turbulence: an invariant dynamical systems approach. J. Fluid Mech., 227:245-272, 1991.

K. Stawiarski and K. Hanjalic. A two-scale second-moment one-point turbulence closure. In Engineering Turbulence Modelling and Experiments - 5, Mallorca, Spain, 2002.

K. Stawiarski and K. Hanjalic. On physical constrains for multi-scale turbulence closure models. Progress in Computational Fluid Dynamics, 5:120-135, 2005.

H. Tennekes and J.L. Lumley. A First Course in Turbulence. The MIT Press, 1972.

H. J. Tucker and A. J. Reynolds. The distortion of turbulence by irrotational plane strain. $J$. Fluid Mech., 32:657-673, 1968.

D. C. Wilcox. Multiscale model for turbulent flows. AIAA Journal, 26:1311-1320, 1988. 


\section{Nomenclature}

\section{Roman Symbols}

$C_{P_{1}} \quad$ Coefficient of the source term in the $\varepsilon_{P}$ equation

$C_{P_{2}} \quad$ Coefficient of the sink term in the $\varepsilon_{P}$ equation

$C_{T_{1}} \quad$ Coefficient of the source term in the $\varepsilon_{T}$ equation

$C_{T_{2}} \quad$ Coefficient of the sink term in the $\varepsilon_{T}$ equation

$c_{\mu} \quad$ Coefficient in the eddy viscosity expression

$f \quad$ Frequency of oscillation

$\mathrm{H} \quad$ Step height in backward facing step flows

$k \quad$ Total turbulent kinetic energy

$k_{P} \quad$ Turbulent kinetic energy stored by the large scales of motion

$k_{T} \quad$ Turbulent kinetic energy stored by the small scales of motion

$\mathrm{K} \quad$ Acceleration parameter in FPGBL cases, $K=\frac{\nu}{U_{\infty}^{2}} \frac{d U_{\infty}}{d x}$

$P_{k} \quad$ Turbulent kinetic energy production rate, $P_{k}=-\overline{u_{i} u_{j}} \frac{\partial U_{i}}{\partial x_{j}}$

$r \quad$ Radial distance

$R \quad$ Radius

Re Reynolds number

$S \quad$ Normal strain in the normally strained flows, $S=\frac{d W}{d z}=-\frac{d V}{d y}$

$S_{i j} \quad$ Mean strain rate tensor, $S_{i j}=\frac{\partial U_{i}}{\partial x_{j}}+\frac{\partial U_{j}}{\partial x_{i}}$

St Strouhal number, $S t=\frac{f \ell}{U_{b}}$

$t \quad$ Time

$T \quad$ Period, $T=\frac{1}{f}$

$U, V, W$ Mean velocity components

$u, v, w \quad$ Fluctuating velocity components

$u_{i}, u_{j} \quad$ Fluctuating velocity components

$u^{\prime}, v^{\prime}, w^{\prime} \quad$ rms of the fluctuating velocity, $u^{\prime}=\sqrt{\overline{u^{2}}}$; in periodic flows: $u^{\prime}=\sqrt{\left\langle u^{2}\right\rangle}$

$\overline{u_{i} u_{j}} \quad$ Reynolds stress tensor

$W_{i j} \quad$ Vorticity tensor, $W_{i j}=\frac{\partial U_{i}}{\partial x_{j}}-\frac{\partial U_{j}}{\partial x_{i}}$

$x, y, w \quad$ Coordinate directions

$x_{i}, x_{j} \quad$ Coordinate directions

$X_{R} \quad$ Time-averaged reattachment point in backward facing step flows 


\section{Greek Symbols}

$\varepsilon \quad$ Turbulent kinetic energy dissipation rate

$\varepsilon_{P} \quad$ Energy transfer rate between the large and small scales of motion

$\varepsilon_{T} \quad$ Energy transfer rate between the small scales of motion and the dissipation zone, thus treated as the turbulent kinetic energy dissipation rate, $\varepsilon_{T}=\varepsilon$

$\phi \quad$ Phase shift in oscillatory flows.

$\rho \quad$ Density

$\nu \quad$ Kinematic viscosity

$\nu_{t} \quad$ Kinematic eddy viscosity

$\sigma \quad$ Turbulent Prandtl number

\section{Superscripts}

+ Non-dimensionalized with inner velocity $U_{\tau}=\sqrt{\frac{\tau_{w}}{\rho}}$

\section{Acronyms}

EXP Experimental data

HLS Hanjalic et al.'s linear-eddy-viscosity two-time-scale model

MTS Multiple Time Scale

SSG Speziale et al.'s Reynolds-stress-transport model

STS Single Time Scale

TKE Turbulent Kinetic Energy 


\section{Appendix - Resume of NT1 and NT2 models}

For the sake of completeness the set of equations and coefficients of both NT1 and NT2 models are presented below.

\section{The NT1 model}

The NT1 model calculates the Reynolds stresses through the linear-eddy-viscosity scheme given by:

$$
\overline{u_{i} u_{j}}=-\nu_{t}\left(\frac{d U_{i}}{d x_{j}}+\frac{d U_{j}}{d x_{i}}\right)+\frac{2}{3} k \delta_{i j}
$$

where the eddy viscosity $\nu_{t}$ is defined as:

$$
\nu_{t}=c_{\mu} \frac{k k_{P}}{\varepsilon_{P}}
$$

where $k$ stands for the total turbulent kinetic energy and is decomposed into $k_{P}$ and $k_{T}$ which stands for the part of the total turbulent kinetic energy which is stored in the large eddies in the production zone and in the smaller eddies in the transfer zone of the turbulent kinetic energy spectrum respectively. They are all related by the expression:

$$
k=k_{P}+k_{T}
$$

The transport equations for the NT1 model are presented below:

$$
\begin{gathered}
\frac{D k_{P}}{D t}=P_{k}-\varepsilon_{P}+\frac{\partial}{\partial x_{j}}\left[\left(\nu+\frac{\nu_{t}}{\sigma_{k_{P}}}\right) \frac{\partial k_{P}}{\partial x_{j}}\right] \\
\frac{D k_{T}}{D t}=\varepsilon_{P}-\varepsilon_{T}+\frac{\partial}{\partial x_{j}}\left[\left(\nu+\frac{\nu_{t}}{\sigma_{k_{T}}}\right) \frac{\partial k_{T}}{\partial x_{j}}\right] \\
\frac{D \varepsilon_{P}}{D t}=C_{P 1} P_{k} \frac{\varepsilon_{P}}{k_{P}}-C_{P 2} \frac{\varepsilon_{P}^{2}}{k_{P}}+\frac{\partial}{\partial x_{j}}\left[\left(\nu+\frac{\nu_{t}}{\sigma_{\varepsilon_{P}}}\right) \frac{\partial \varepsilon_{P}}{\partial x_{j}}\right] \\
\frac{D \varepsilon_{T}}{D t}=C_{T 1} \frac{\varepsilon_{P} \varepsilon_{T}}{k_{T}}-C_{T 2} \frac{\varepsilon_{T}^{2}}{k_{T}}+\frac{\partial}{\partial x_{j}}\left[\left(\nu+\frac{\nu_{t}}{\sigma_{\varepsilon_{T}}}\right) \frac{\partial \varepsilon_{T}}{\partial x_{j}}\right]
\end{gathered}
$$

The coefficients of the NT1 model are presented in Table 5 where:

$$
\begin{gathered}
\eta=\max \left[\frac{k}{\varepsilon_{T}} \sqrt{\frac{1}{2} S_{i j} S_{i j}}, \frac{k}{\varepsilon_{T}} \sqrt{\frac{1}{2} W_{i j} W_{i j}}\right] \\
S_{i j}=\frac{\partial U_{i}}{\partial x_{j}}+\frac{\partial U_{j}}{\partial x_{i}} \\
W_{i j}=\frac{\partial U_{i}}{\partial x_{j}}-\frac{\partial U_{j}}{\partial x_{i}} \\
f_{B L}=\left(\frac{k_{P}}{k_{T}}\right)_{B L}\left(1-f_{1}+f_{2}\right)=3.6\left(1-f_{1}+f_{2}\right) \\
f_{1}=1.7\left(\frac{k_{P}}{k_{T}}-1\right) \min \left(\frac{P_{k}}{\varepsilon_{T}}-1.9,0\right) \max \left(\frac{\varepsilon_{P}}{\varepsilon_{T}}-1,0\right)
\end{gathered}
$$




$$
\begin{gathered}
f_{2}=20 \max \left[\frac{k_{P}}{k_{T}} \max \left(\frac{\varepsilon_{P}}{\varepsilon_{T}}-1.1,0\right) S W, 0\right] \\
S W=\frac{S_{i j} S_{i j}-W_{i j} W_{i j}}{S_{i j} S_{i j}+W_{i j} W_{i j}}
\end{gathered}
$$

The expression for the dimensionless shear $\eta$ presented in equation 53 is the general expression proposed by Craft et al. (1996) which reduces to $\frac{k}{\varepsilon_{T}} \frac{d U}{d y}$ in simple shear flows.

\begin{tabular}{|c|c|c|c|c|c|c|c|}
\hline$c_{\mu}$ & & $C_{P 1}$ & $\overline{C_{P 2}}$ & $\sigma_{\varepsilon_{P}}$ & $C_{T 1}$ & $\overline{C_{T 2}}$ & $\sigma_{\varepsilon_{T}}$ \\
\hline $\min \left[0.115,0.023+0.25 e^{-0.30 \eta}\right]$ & $\max$ & {$\left[1.4921+2.5 \frac{\min \left(0, \frac{k_{P}}{k_{T}}-f_{B L}\right)}{\frac{k_{P}}{k_{T}}+f_{B L}}, 0\right]$} & 1.8 & 1.4202 & 1.6 & 1.7 & 1.2181 \\
\hline
\end{tabular}

Table 5: Coefficients of the NT1 model

And finally, the asymptotic states predicted by the NT1 model are presented in Table 6 below.

Table 6: Values expected to be provided by the NT1 model in the asymptotic states studied

\begin{tabular}{cccccccc}
\hline \hline$\left(\frac{P_{k}}{\varepsilon_{T}}\right)_{S F}$ & $\left(\frac{k_{P}}{k_{T}}\right)_{S F}$ & $\left(\frac{\varepsilon_{P}}{\varepsilon_{T}}\right)_{S F}$ & $\left(\frac{k_{P}}{k_{T}}\right)_{D F}$ & $\left(\frac{\varepsilon_{P}}{\varepsilon_{T}}\right)_{D F}$ & $\left(\frac{k_{P}}{k_{T}}\right)_{B L}$ & $\left(\frac{\varepsilon_{P}}{\varepsilon_{T}}\right)_{B L}$ & $c_{\mu_{B L}}$ \\
\hline 1.9 & 4.4 & 1.1667 & 1.0 & 0.5 & 3.6 & 1.0 & 0.115
\end{tabular}

\section{The NT2 model}

The NT2 model calculates the Reynolds stresses through the linear-eddy-viscosity scheme given by:

$$
\overline{u_{i} u_{j}}=-\nu_{t}\left(\frac{d U_{i}}{d x_{j}}+\frac{d U_{j}}{d x_{i}}\right)+\frac{2}{3} k \delta_{i j}
$$

where the eddy viscosity $\nu_{t}$ is defined as:

$$
\nu_{t}=c_{\mu} \frac{k^{2}}{\varepsilon_{T}}
$$

where $k$ stands for the total turbulent kinetic energy and is decomposed into $k_{P}$ and $k_{T}$ which stands for the part of the total turbulent kinetic energy which is stored in the large eddies in the production zone and in the smaller eddies in the transfer zone of the turbulent kinetic energy spectrum respectively. They are all related by the expression:

$$
k=k_{P}+k_{T}
$$

The transport equations for the NT2 model are presented below:

$$
\begin{gathered}
\frac{D k_{P}}{D t}=P_{k}-\varepsilon_{P}+\frac{\partial}{\partial x_{j}}\left[\left(\nu+\frac{\nu_{t}}{\sigma_{k_{P}}}\right) \frac{\partial k_{P}}{\partial x_{j}}\right] \\
\frac{D k_{T}}{D t}=\varepsilon_{P}-\varepsilon_{T}+\frac{\partial}{\partial x_{j}}\left[\left(\nu+\frac{\nu_{t}}{\sigma_{k_{T}}}\right) \frac{\partial k_{T}}{\partial x_{j}}\right] \\
\frac{D \varepsilon_{P}}{D t}=C_{P 1} P_{k} \frac{\varepsilon_{P}}{k_{P}}-C_{P 2} \frac{\varepsilon_{P}^{2}}{k_{P}}+C_{P 1}^{\prime} k_{P} \frac{\partial U_{l}}{\partial x_{m}} \frac{\partial U_{i}}{\partial x_{j}} \epsilon_{l m k} \epsilon_{i j k}+\frac{\partial}{\partial x_{j}}\left[\left(\nu+\frac{\nu_{t}}{\sigma_{\varepsilon_{P}}}\right) \frac{\partial \varepsilon_{P}}{\partial x_{j}}\right]
\end{gathered}
$$




$$
\frac{D \varepsilon_{T}}{D t}=C_{T 1} \frac{\varepsilon_{P}^{2}}{k_{T}}-C_{T 2} \frac{\varepsilon_{T}^{2}}{k_{T}}+\frac{\partial}{\partial x_{j}}\left[\left(\nu+\frac{\nu_{t}}{\sigma_{\varepsilon_{T}}}\right) \frac{\partial \varepsilon_{T}}{\partial x_{j}}\right]
$$

The coefficients of the NT2 model are presented in Table 7 where:

$$
\begin{gathered}
\eta=\max \left[\frac{k}{\varepsilon_{T}} \sqrt{\frac{1}{2} S_{i j} S_{i j}}, \frac{k}{\varepsilon_{T}} \sqrt{\frac{1}{2} W_{i j} W_{i j}}\right] \\
S_{i j}=\frac{\partial U_{i}}{\partial x_{j}}+\frac{\partial U_{j}}{\partial x_{i}} \\
W_{i j}=\frac{\partial U_{i}}{\partial x_{j}}-\frac{\partial U_{j}}{\partial x_{i}} \\
f_{B L}=\left(\frac{k_{P}}{k_{T}}\right)_{B L}\left(1-f_{1}+f_{2}\right)=3.7\left(1-f_{1}+f_{2}\right) \\
f_{1}=5.4\left(\frac{k_{P}}{k_{T}}-1.3166\right) \min \left(\frac{P_{k}}{\varepsilon_{T}}-1.9,0\right) \max \left(\frac{\varepsilon_{P}}{\varepsilon_{T}}-1,0\right) \\
f_{2}=11 \min \left\{1.8, \max \left[\frac{k_{P}}{k_{T}} \max \left(\frac{\varepsilon_{P}}{\varepsilon_{T}}-1,0\right) S W, 0\right]\right\} \\
S W=\frac{S_{i j} S_{i j}-W_{i j} W_{i j}}{S_{i j} S_{i j}+W_{i j} W_{i j}}
\end{gathered}
$$

The expressions for $\eta, S_{i j}, W_{i j}$ and $S W$ are the same for the NT1 model, however they are being repeated here in order to present the full version of the NT2 model.

Table 7: Coefficients of the NT2 model

\begin{tabular}{cccc}
\hline \hline$c_{\mu}$ & $C_{P 1}$ & $C_{P 2}$ & $\sigma_{\varepsilon_{P}}$ \\
\hline $\min \left[0.09,0.0117+0.22 e^{-0.31 \eta}\right]$ & $\max \left[1.5697+2.5 \frac{\min \left(0, \frac{k_{P}}{k_{T}}-f_{B L}\right)}{\frac{k_{P}}{k_{T}}+f_{B L}}, 0\right]$ & 1.8 & 1.6664 \\
\hline$C_{T 1}$ & $C_{T 2}$ & $\sigma_{\varepsilon_{T}}$ & $C_{P 1}^{\prime}$ \\
\hline 1.0 & 1.1 & 1.1922 & -0.005 \\
\hline
\end{tabular}

And finally, the asymptotic states predicted by the NT2 model are presented in Table 8 below.

Table 8: Values expected to be provided by the NT2 model in the asymptotic states studied

\begin{tabular}{ccccccccc}
\hline \hline$\left(\frac{P_{k}}{\varepsilon_{T}}\right)_{S F}$ & $\left(\frac{k_{P}}{k_{T}}\right)_{S F}$ & $\left(\frac{\varepsilon_{P}}{\varepsilon_{T}}\right)_{S F}$ & $\left(\frac{k_{P}}{k_{T}}\right)_{D F}$ & $\left(\frac{\varepsilon_{P}}{\varepsilon_{T}}\right)_{D F}$ & $\left(\frac{k_{P}}{k_{T}}\right)_{B L}$ & $\left(\frac{\varepsilon_{P}}{\varepsilon_{T}}\right)_{B L}$ & $c_{\mu_{B L}}$ & $c_{\mu_{S F}}$ \\
\hline 1.9 & 8.8245 & 1.0916 & 1.3166 & 0.5683 & 3.7 & 1.0 & 0.09 & 0.0336
\end{tabular}

